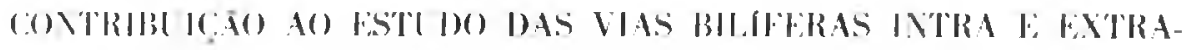 \\ HEITIUCAS NO FEUS MO.MESTICA \\ SISTHUATHA(AO) DO RAILS PRINCIPAIS DEITER

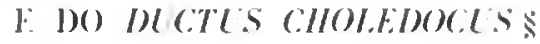

Antonio FERNANDES FILHO *

\begin{abstract}
RFMIV'A II

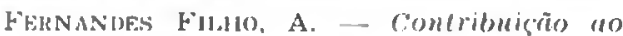
estudo dess riess biliforess intre extrehevatices no Felis domestica. Sistemetizreguo do ramus principalis dexter e do ductus choledocus. Kev. Fac. Med. vet.
\end{abstract} Zootece. Iniv. S. I'aulo, 11): 79-102, 1973.

REST:MO - A sisfematizacio do ramus principalis dexter e do ductus choledocus motivo destu pesquisu, foi estudadu cm jo fígudos de gutos.

Baseados ma dissecrão destes irgrous podrmos informar ane o ductus choledorus resulte:

a) e'm 3.t peross $(68,0 \%)$, der fusion do ramus principalis dexter a damus principulis sinister:

b) em 11 cossos (22.0\%), des renuirio do ductus hepaticus en ductus ('ysticus;

(c) em is propureceres $(6,0 \%)$, da triplice conflurnein do ramus principalis dexter. ramus principalis sinister e ductus cysticus;

d) $\mathrm{em} 1$ dissecruo $(2.0 \%)$, da triplice romeryrnciu do ramus principalis dexter. ramus principalis sinister e ranus medialis lobi dextri;

e) em I figudo (2.0\%), du juncrio do ductus hepaticus e ramus lobi quadrati.

No que tronge to sistema do ramus principalis dexter. conforme verifirumos. mos tra-st: constifuido pela associegüo dos seguintes ductos ronspicuos: ramus latcralis lobi dextri. ramus processi caudati, ramus medialis lobi dextri, ramus processi papillaris, ramus lobi quadrati e ductus cysticus.

UNITERMOS - Vias biliferes"; Gutos*.

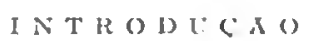

(os poucos e resumirlos compêndios rledicados, particularmente, ao esturlo da Anatomia do gato doméstico c as informacōes neles colhidas. com referência às vias bill. feras intra e extra-hepáticas. expostas $\mathrm{cm}$ escassas linhas de conteúrlo impreciso, levaram-nos a ajuizar que, sobre tal matéria, a ninguém aproveita consultá-los. De outro larlo, como logo a seguir se verá. os darbs of rereidos por AA. de trabathos especializados, embora proporcionando-nos conhecimentos bem mais precisos a resporito do sistema do drenagem do figado desse animal, estão ainda longe de retratar as múltiplas modalidades do associação dos seus coletores, se'jam em virturle do reduzido numern de pecas utilizado para fundamentar as conclusōes, sẹja face ao critério imprimido à exposiçăo dos resultados.

A investigação que emprerndemos e agora divulgamos filia-se a una das linhas de pesquisa em curso na Cadeira de Anatomia da Faculdade de Medicina Veterinária da Universidade de São Paulo e tem por objetivo sanar, parcialmente, na medida do possivel, as falhas apontadas. Assim. atribuimo-nos a tarefa de examinar o arranjo dos ductos biliferos responsáveis pela constituiçäs do rumus principalis dexter a

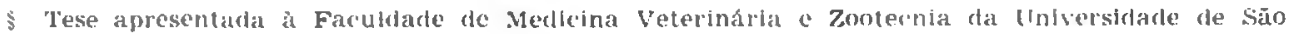
Paulc.

* Professor Assistente Doutor.

Departamento de Cirurgia e obstetricia da Faculdade de Medicina veterinaria e Zootecnia da I'.S.P. 
FERNANDES FILHO. A. - Contribuição an estudo das vias biliferas intra e extra-hepáticas no Felis domesfica. Sistematizacão do remus principalis dextêp e do ductus choledocus,

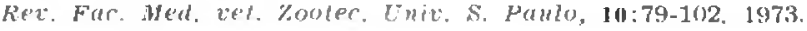

ductus choledorus. sem descuidar do comportamento do durtus cysticus. valendo-nos, para tanto, dos métodos de dissecceâo e radiológien.

\section{I $\mathrm{T}$ \& $\mathbb{R}$ A $\mathrm{T}$ L $R A$}

OTTAVIANI" (1933), realizando estudo radiográfico comparativo das vias biliferas intra-hepáticas. identifica. genericamente, dois territórios, um direito e outro esquerdo. determinados por plano sagital que passa pelo ponto de encontro das referidas vias, ou srja, na metare direila da fossa transversa. No que respeita ao gato doméstico, táis territórios, cada qual com seu ducto, não mostram anastomoses entre si. à custa de afluentes primários ou sccundários. Fxcepcionalmente, afluente primário, oriundo de parto do lobo quadrado. integrante do territorio esquerdo. corro para a direita. O territirio direito, porém, porte apresentar dois condutos, um do lobo direito e um do lobo caudato. Comumente. os afluentes primários do lobo direito unem-se formando coletor onde desemiuca o pertencente ao lobo caudato. Algumas vezes, entretanto, este lobo permanece independente e constilui via tributária do ducto hejático. A parte esquerda do lobo caudato exibe fino afluente primário, tortuoso, que vai terminar no conduto bilifero esquerdo ou, rliretamente, no ducto hepático.

Radlografia das vias biliferas do gato. injetadas com substância radiopaca, acompanha o trabalho.

BRLSSOU \& VLADUTIU 2 (194.4), esludando a distribuiçāo das artérias, velas c canais biliares, $\mathrm{cm} 32$ figados de gatos domésticos, de idades e lalhes diversos, mediante o emprego do dissecçōes, corrosōes o radiografias, esclarecem que as vias biliferas unem-se gradativamente, em scu trajeto para constituirem, as nivel do hilo, dois troneos principais: o (anal hepático esquerdo o o canal hepático direito. Este. bon menor, resulla da junção de dois grandes troncos. () tronen superior coleta, por várias rázes, a bile do lobo caudato e do lobo direito; o tronco inferior, muito mais longo, percorre o lobo intormodiário direito. a todo o comprimento, dele recebendo numerosos afluentes. No hilo, os dois canais hepáticos fundem-se $e_{\text {, }}$ com o dueto cistico. que chega ao ângulo de convergência dos citados canais, dão origom ao colédoco. $K \dot{m}$ definitivo, as ra. mificaçôns da veia porta. da arteria hepática e dos canais biliares parecem distribuir-se segundo plano análogo, composto de dois territórios, um direito e outro esquerdo.

'Três figuras esquemáticas ilustram a disposição clas artérias, veias e canais biliferos. (m) scparado.

NETTEI,BIAD * (1954), pesquisando 56 figados de diforentes mamiferos. entre os quais os de 11 gatos. serve-se de 7 peças para exame da ramificação do ductus heputirus. Após a injeção dos órgẫos com massa destinada à ubtenção de preparaçōes por corrosĩo, o A. chrgou aos seguintes resultados:

a) em 6 dos 7 casos o ductus heputicus divide-se em dois ramos, ou seja, no ramus mincipalis dexter e no rumus mincipalis sinister;

b) cm 1 peça o ductus hepaticus trifurca-se, dando o rumus principulis dexter, o ramus ventrocranialis dexter o o ramus principalis sinisfer;

c) o rumus ventrocrmialis dexter, em 5 dos casos, emerge do ramus principalis dexter e, 1 vez, do ramus principalis sinister:

d) do ramus pincipulis dexter originamse o romus dorsoraudalis dexter e o rumus dorstulis dexter;

e) o durtus cysticus dosemboca, en 3 preparaçóes, no ramus ventrocranialis dexter; em 2 peças, no rumus principulis dexter; 1 vez no rumus principalis sinister e, 1 vez, no tronco do ductus hepalicus.

O A. oferecen quatro esquemas relativos ao comportamento da venu portue (lo ductus hejuticus do gato. 
FERNANIDES FILIO, A. - Contribuicáo an estudo das vias biliferas intra e extra-hepaticas no Felis domestica. Sistematizacăo do romus principalis dextep (s do durtus cholederus.

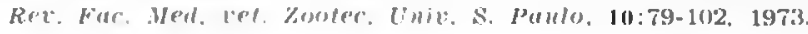

JABLAN-PANTIC: (1963), estudando as vias biliferas intrahepaticas, $\mathrm{cm} 58$ animais domésticos. dos quais 5 gatos ${ }^{\circ} 7$ cães de ambos os sexos e de diferentes raças e idarles, após injeção dos fígados com substância radiopaca. através do ductus choletocus, seguida de fixação pela formalina a $4 \%$. oferece relato único para ambas as espécies; suas conclusões, assim se sintetiram:

a) o ductus heputions fornece dois ramos, isto é, o rumus principulis dexter co rumus principulis sinister;

b) o ramus principulis dexter drena o lobus lateralis dexter, o processus caudutus do lobus cundatus e, o lobus mediulis dexter, por intermédio, respectivamente, do rumus luterulis lobi dextri, rumus processi canduti e rumus medialis lobi dextri;

c) o rumus luteralis lobi dextri a o ramus mediatis lobi dextri desembocam. isoladamente, no ramus principulis dexter;

d) no gato, especificamente, chegam, às veres. ao rumus principalis dexter, o rumus lobi auadrati o o rumus medialis lobi sinistri;

(2) o ramus medialis lobi dextri abre-se, com maior freqüência, no ductus cysticus:

f) o rumus proxessi couduti. em certos casos, recebe ramo secundário do processus pupilluris; por vezes, este vai diretamente ao ductus choledocus;

g) o ductus cysticus lança-se, mais comumente, no ductus heputicus mas, pode atingir também, o ramus mincipalis dexter, o ramus principulis sinister ou o ramus inediulis lobi dextri.

O A. documenta os resultados de sua investigação, no que se refere an gato, com quatro esquemas e um colecistograma.

Passemos agora ans resumos das informaçōes colhidas nos tratadistas, cuidem eles, genericamente. dos animais domésticos ou, especificamente, do Felis domestica.

LESBRE " (1922) limita-se a relatar que o gato apresenta canais hepato-cisticos.
MAR'TIN (1923) descreve que dos lobos hepáticos procedem 8 a 10 duclus biliferi. reunidos em 3 a 5 calibrosos ductus heputici distintos. Estes abrem-se, separadamente, em parte no durtus cysticus, como ductus hepatocystici c em parte no prolongamento do ducto principal que, a partir da abertura do último ductus hejeticus. poderia ser designado ductus choledochus.

ELLENBF:RGER \& BALM ' (1932) afirmam que dos lobos hepáticos. originam-se 8 a 10 durtus biliferi, os quais se fundem ('m 3 a 5 mais conspicuos ductus heputici; estes últimos abrom-se separadamente no ductus cysticus e, a partir da abertura do último deles, recobe o nome de ductus choledorhus.

RFIGHARD \& JENNINGS 10 (1935) informam ser o ducto cistico, alcançado, na sua extremidade distal, por dois ou mais ductos hepáticos. A relação de tais ductos $\mathrm{com}$ o cistico varia, ou seja, cles porem atingi-lo por tronco comum ou separadamente. Destes ductos hepáticos, um resulta da junção de ductos menores das metades esquerdas do figado e do lobo cistico, enquanto o outro forma-se. analogamente, pela uniāo de pequenos ductos da metarle direita do lobo cístico, de ambas as divisões do lobo lateral direito e do lobo caudato. O ducto originado da fusão dos ductos hepáticos e cístico denomina-se ducto biliar comum (ductus communis choledochus).

DAVISON 3 (1947) refere que a bile pas. sa por numerosos ductos oriundos dos vários lobos, rumo ao ducto hepático principal, visivel do lado direito da vesícula biliar. O ducto cistico mostra-se relativamente flexuoso. O ducto hepático principal alcança o cístico mais ou menos a mrio comprimento e, o ducto assim formado. é conhecido como ducto biliar comum (ductus choledocus).

BOURDELLE \& BRESSOU ' (1953) inrlicam que o aparelho excretor biliar do cão e gato compreende o canal do colédoco, formado pela união de alguns canais hepáticos e o canal cistico. Este alcaņ̧a o canal 
FFINANDES FILIIO, A. - Contribuicão au estudo das vias biliferas intra e cextra-hepaticas

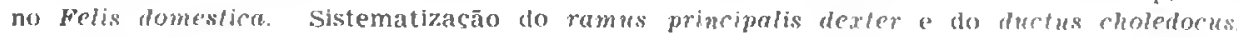
Rev. Fuc. Med. vet. Kootec. Lniv. S. Paulo, 10:79-102. 1973.

hepático no centro do figado. não sem antes ter recebido, sob o nome de canais hepato-císticos, coletores vindos diretamente do parênquima hepático. O canal hepático não é único mas, rèpresenta sistema de canais, um ou dois para cada lobo, que vāo atingir. isoladamente, o canal cístico. () canal coledoco trm a mesma direção que os canais hepáticos: muito curto, segue trajeto retilineo, do hilo ao duodeno e acothe o canal excretor próprio do lobo direito que nele se lanca, separadamente. perto de sua terminação.

\section{MATHRIAI, F: METODO}

Nossas observacôns esteiam-se no estudo de 50 peças, compreenciendo, cada uma, fígado e trato duodenal. retirados $\mathrm{em}$ bloco, de gatos domésticos, 29 machos 123 adultos e 6 jovens) e 21 fêmeas (17 adultas e 4 jovens). de raças não definidas, recolhidos pela Faculdade de Medicina Veterinária da Universidade de São Paulo.

Isolado o conjunto, convenientemente, abrimos o duodeno ao longo da borda livre e, exposta a mucosa. canalizamos o ducto coléctoco através da jupilla duodeni major (papila duodenal maior), esvasiando, entāo, o sistema bilifero, mediante suaves massagens a compressões; injetamo-lo a seguir, pela mesma via, com solução de grlatina a 10\% ( $p / v)$ em água, corada pelo cinábrio, contendo celobar (Laboratório Mauricio Villela) em suspensão a $50 \%$. Os órgãos foram, sucessivamente, radiografados. fixados em formol a $10 \%$ e, dissccados a vista desarmada ou, se necrssário sob lupa, utilizando-se pequenos aumentos; concluida a preparaçāo, colhemos esquemas e fotografias de todas as peças para apreciação final.

Os territórios hepáticos, nesta espécie, excecão feita ao lobus medialis dexter e lobus quadratus (lobo medial direito e lobo quadrado), em parte fundidos, são bom demarcados por profundas incisuras interlobares, em virture do que, a pars communis apresenta-se muito reduzida. Para delimi- tá-los, idealizamos plano imaginário que. disposto perpendicularmente às superficies diafragmática e visceral do figado, corta a fossu vesicue fellene (fossa da vesicula biliar), segundo linha vista a percorre-la no sontido ro maior eixo o a atingir, a impres-

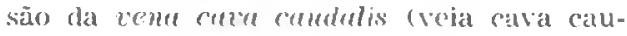
dall.

Na sistematizaçāo das vias biliferas lơnamos em conta, apenas, os territórios escoados e não calibre dos ductos, pelo falo de que as injeçoes cnchem melhor os de situação superficial; tonhat-se. pois, em mente. que empregamos as expressões tributários, afluentes, etc., em termos de associaçāo.

Cumpre esclarecer que, havendo dado realce ans coletores responsáveis pela drenagem de regiōes hepáticas bem definidas, não estendemos nossa atenção. por desnecessário, face aos critérios estabelecidos, à raizes dos ramos principais; $\mathrm{em}$ contraposiçào, sempre assinalamos as contribuições implicadas na combinaçảo das vias de escoamento de territórios distintos, independentemente do calibre, da procedência e destinaçāo delas, anotando. ainda. quando representavam curtos troncos, o respectivo número de componentes.

Convencionamos orientar os relatos no sentido periferia-centro, indicando os ramos anatomicamente diferenciados dos restantes, polas mencionadas caractoristicas, até a sua reuniāo com outros de igual calibre. para compor troneo inominacio.

Adot $r$ mos na apresentaço dos resultados. a nomenclatura enipregada por JABLANPANTIC.

R FS U I. TADOS

Nossos resultados, concernentes à sistematização das vias biliferas no gato do. méstico, dizem respeito a dois itens, ou seja:

A) ductus choledorus; B) momes principrolis dexter. 
FERNANDES FILHO, A. - Contribuicão ao estuclo das vias biliferas intra e extra-hepaticas no Felis domestica. Sistematizaç̆o do ramus principalis dexter e do rurtus rholedorus. R'v. Far. Mell. vet. Zooter. Univ. S. Paulo, 10:79-102, 1973.

\section{A) Ductus choledocius}

Ėm 50 dissecções o rumus principalis dexter e o rumus principalis sinister contribuem, direta ou indiretamente, para a formaçāo do ductus choledocus, que em 34 delas $(68,0 \% \pm 6.6 * \ldots$ Figs. 1, 2, 3) apresenta apenas estas duas raizes. Nos 16 casos restantes $(32,0 \% \pm 6,6)$, vê-se o ductus choledocus, 11 vezes $(22,0 \%$ t. 5,8$)$, a resultar da uniāo do ductus heputicus e ductus cysticus; 3 vezes $(6,0 \% \pm 3,4)$, a nascer da triplice convergência do ramus principulis dexter, ramus principalis sinister e luchus cysticus, 1 vez $(2,0 \% \pm 2,0$ - Fig. 4), a derivar da triplice confluência do ramus principalis dexter. ramus principalis sinister e ramus medialis lobi dextri e 1 vez $(2,0 \% \pm 2,0)$, a surgir da junção do ductus hepaticus e rumus lobi quadruli.

Quando o ductus choledocus é constituido pela fusão do ramus principalis dexter e rumus princiualis sinister, 18 vezes $(36,0 \%$ $\pm 6,8$ - Figs. 1, 2) o ductus cysticus integra-se ao sistema do primeiro e 16 vezes $(32,0 \% \pm 6,6-$ Fig. 3), lança-se no sistema do segundo.

Cabe ainda ressaltar e aduzir que, além de pertencer a estes dois sistemas e de dar origem ao ductus choledocus, 3 vezes $(6,0 \%$ $\pm 3,4$ ), mediante triplice convergência, por associaçāo com o rumus principalis dexter e rumus principulis sinister, em 11 preparaçōes $(22,0 \% \pm 5.8)$ o ductus cysticus aparece como tributário do ductus heqaticus, endereçando-se, $1 \mathrm{vez}$ (Fig. 4) ao ramus medialis lobi dextri e na peça restante, ao ramus lobi quadrati, nos casos $\mathrm{cm}$ que estes dois últimos ramos representam constituintes diretos do ductus choledocus.

Individualizado o ductus choledocus, encontramo-lo, apenas em 1 caso $(2,0 \% \pm 2,0)$, a receber eferente do lobus luterulis sinister.

Desvio padrão.

\section{B) Ramus principalis dexter}

Na maioria das peças, integram o sistema do ramus principalis dexter, o ramus lateralis lobi dextri, o rumus processi caudati, o ramus medialis lobi dextri e, ainda, de modo inconstante, o ramus processi papillaris, o ramus lobi quadrati e, como se disse, o ductus cysticus, além de contribuicoōes oriundas do lobus laterulis dexter, processus cuudutus do lobus caudutus, lobus mediutis dexter, processus papillaris do $70-$ bus cuudutus e lobus cuudrutus.

1. Rumus luteralis lobi dextri e Ramus processi caudati - Nas 50 peças, o ramus luteralis lobi dextri e o rumus processi caudati concorrem para a formaçāo do ramus principulis dexter, sendo a conjugaçāo de ambos uma das mais comuns disposiçōes dos ductos conspicuos vistos a compor seu sistema. De fato, 47 vezes $(94,0 \% \pm 3,4-$ Figs. 1, 2, 3, 4) tais ramos concorrom para a formação da raiz mais regularmente organizada do rumus principalis dexter. Esta raiz é encontrada, em 31 peças $(62,4 \% \pm 6,9)$, a surgir da junçāo de ductos que drenam, exclusivamente, os respectivos territórios glandulares, vale dizer, o lobus lateralis dexter e o processus caudatus do lobus cuudalus; nas mais $16(32,0 \% \pm 6,6)$, ela mostra-se constituída pela uniāo dos mesmos ramos, mas estes acolhem, sob diferentes combinaçōes, coletores de outras regiōes. Assim, em 13 figados $(26.0 \% \pm 6,2)$, somente o ramus processi caudati recebe eferentes:

a) apenas do lobus lateralis dexter, 9 vezes, a saber, um colctor - 7vezes; dois ductos -2 vezes; elucide-se que no referente a 1 das peças, a contribuição representa tronco de dois componentes;

b) unicamente do processus papillaris do lobus caudatus, mediante tronco formado por dois contingentes -2 vezes; 


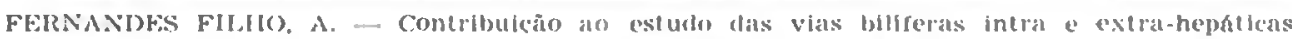
no Felis domestica. Sistematizacano do rumus primcipalis dester e do ductus choledocus.

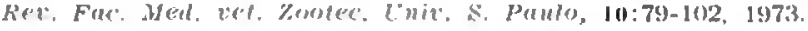

c) simultancamente do lobus latemlis derter c do processus pripilleris do lobus curudutus, por intermédio de troneo resultante da fusáo de dois rluctos - 2 vezes (Fig. 2).

Ëm 3 dissecçōes $(6.0 \%$ 上 3,4$)$, apenas b) romus luterolis lobi dextri acolhe oferentes:

a) somente do processus caudatus do lobus caudatus. uma contribuição - 2 veres;

b) exclusivamente do lobus modialis dexter. dois coletores - 1 ve\%.

A via bilifera composta pelo remus laterulis lobi dertri o rumus processi conduli, que o identificada lisre de tributários 21 vezes $(42,0 \% \pm 7.0$ - Figs. 2, 3, 1). nos mais 26 casos $(52.0 \%$ a. 7.1$)$ acolhe rluctos escoadores:

a) simultaneamente do lobus loteralis dexlev a processus rumdatus do lobus coudulus. 7 vezes, ou seja, dois eferentes cuja chegada se dá por ordem inversa à da enumeração dos lobos - 2 vezes; contingente originack do primeiro território e duas contribuiçoes do segundo - 1 vez, coletor procedente deste. intermediário a dois eferentes oriundos daquele - 1 vez; tronco integrado por um contingonte de carla região -2 vezes; tronco constituido por duas contribuiçós do fobuss luterelis dexter, colocarlo antes de coletor do processus candeatus do lobus crendulus - 1 ver;

b) unicamente do processus crudulus do lobus cumblus. 6 vezes ou, mais exatamente, um eferente - 5 vezes; dois contingentes - $1 \mathrm{v} \%$; noto-se que $\mathrm{cm}$ 2 das preparaçōes. o eferente corresponde a troneo para o qual concorrem três coletores:

c) apenas do lolus lulcrulis dexter, uma

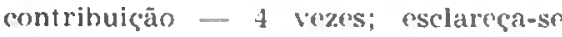
que 'm 1 das disseccōes. cla surge como troneo de dois componentes;

(1) simultaneamente do lobus lutfrulis dexter. do processiss crmedulus do lobus rendulus o lobus mediulis dexter, 3 vozes ou, melhor precisando, eferente do primeiro território. matis tronco que reune duas contribuiçōes do segundo ea colotor do toreciro - 1 vez; contingente do processus crublalus do lobus condutus, outro do lobus lateralis dexter e tronco que conjuga três cferentes do lobus mediulis dexter - 1 vez; via composta por duas contribuiçoes oriundas, respectivamente, da primeira é tercrira ciladas regióes, mais coletor independente da segundal - 1 ver:

c) simultaneamente do proressues couclutrs e processus papilloris do tobus rem-

Figs. (1 a t) representalivos das vias bilferas intra e extra-hepatloas no Felis tome'sfica

$$
\begin{aligned}
& \text { c- ancius rholedacus } \\
& \text { d - ramus principalis aneser } \\
& \text { c- ramus principalis sinisler }
\end{aligned}
$$

1 - ramus lateralis lobi dertri

I' - contrlbuicảo do lobus lateralis dexter

2 - ramus proressi camalati

3 - ramus medialis lobi derfri

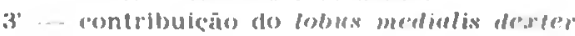

1 - ramus processi papillaris

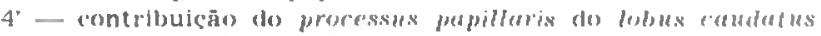

5 - ramus lobi quadrali

fi - duetus ryspirus 
FFIRNANDES FILIIO, A. - Contribujeăo ao estudo das vias biliferas intra extra-hepalicas

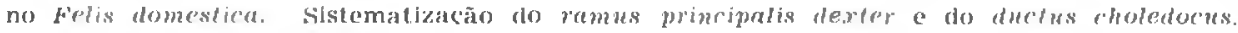
herl. Fuc. Hed, vet. Zontec. Iniv. S. Pavlo, 10:79-102, 1973.

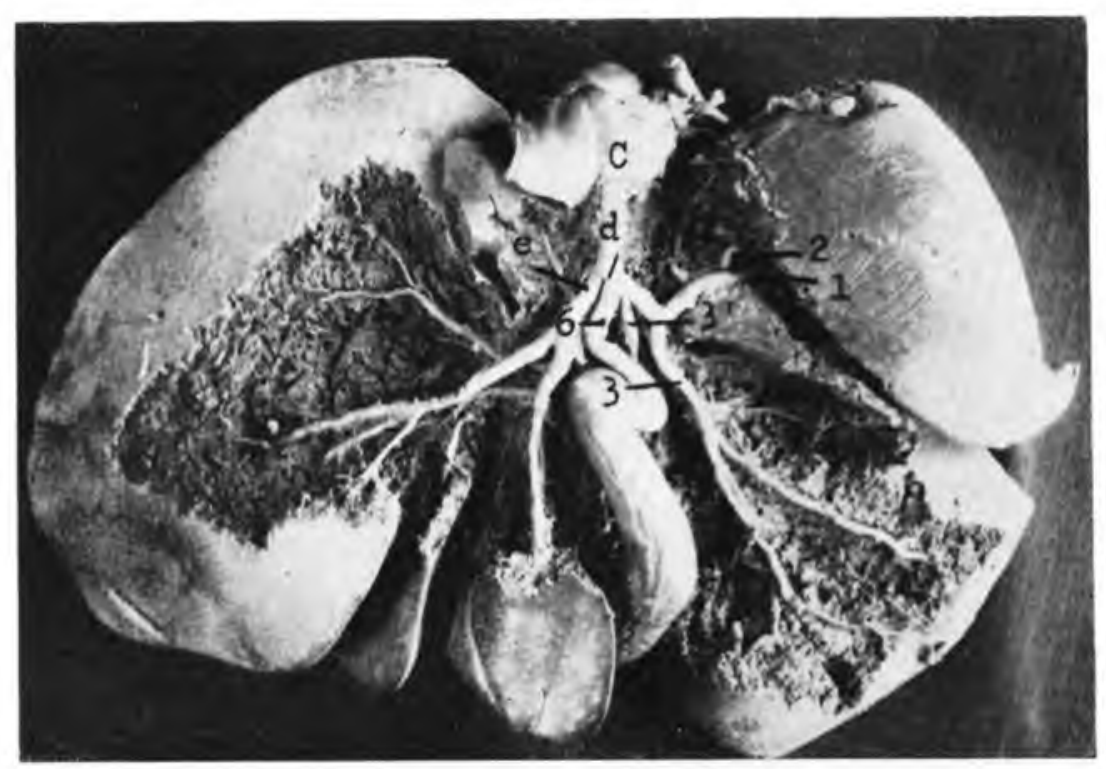

Fig. 1

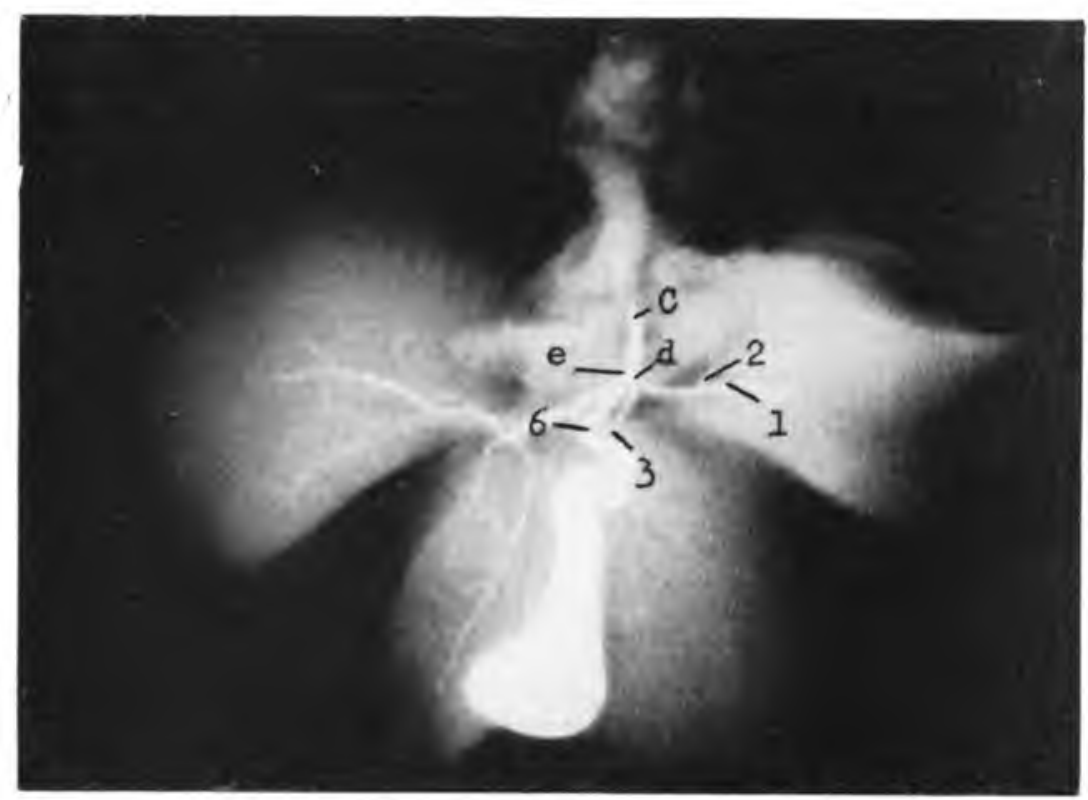

Fig. 2 
FERNANDES FILII), A. - Contribuicũo an estucle das vias biliferas intra e extra-hepáticas no felis domestira. Sistematizacão do ramus principalis dexter e cho durfus choledocus.

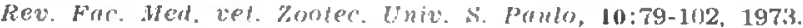

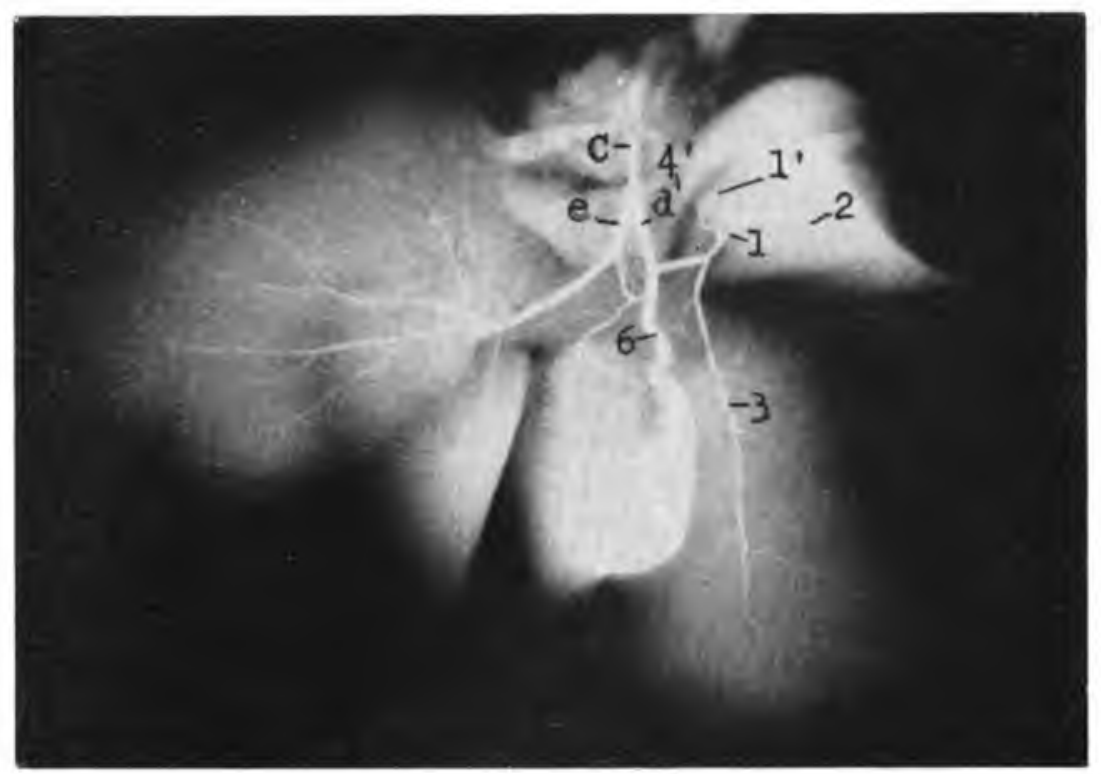

Fig. 3

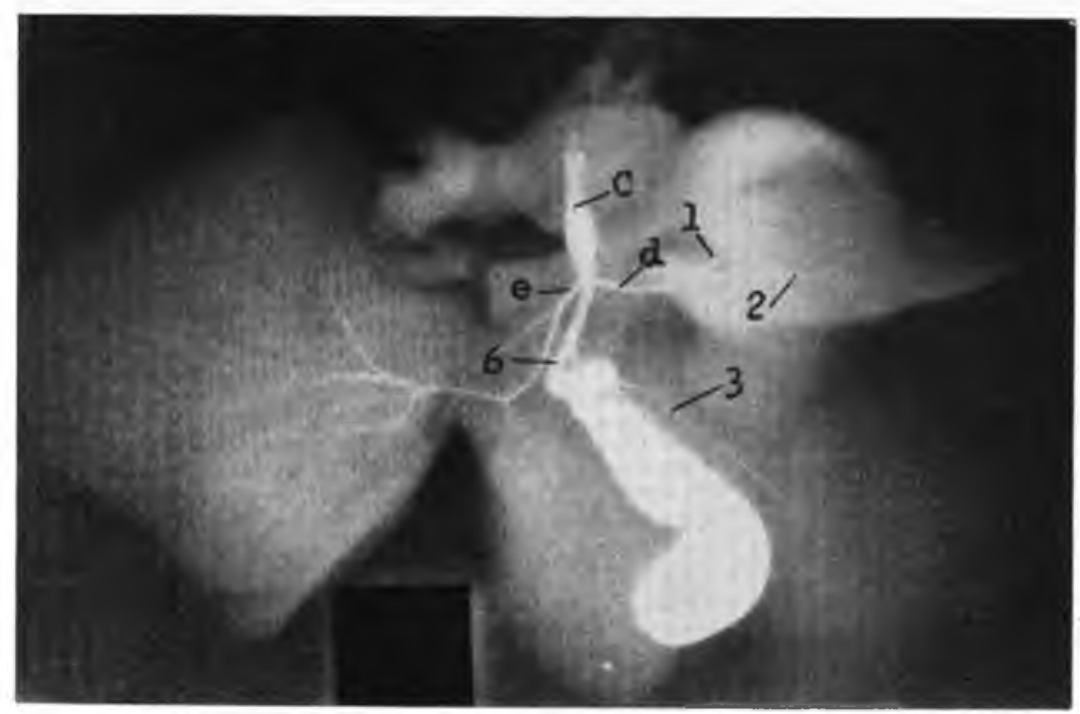

Fig. . 
FFR.NAXDFs FILIIO, A. - Contribuicão ao estudo das vias billferas intra e extra-hepditleas no Felis domesficu. Sistematizaçăo do ramus principalis dexter c do ductus choledocus.

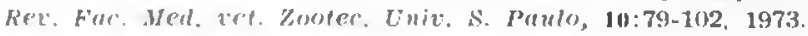

dulus, 2 vezes, vale dizer, um eferente de cala território - $1 \mathrm{vez}$; tronco para o qual eles afluem - 1 vez;

f) somente do lobus medialis dexter, ou seja, tronco integrato por dois contingentes - 1 vez;

g) ao mesmo tempo do processus caudatus do lobus coudutus e lobus medialis dexter ou, mais exatamente, fronco constituido por uma contribuição de cacla regiāo … 1 vèz;

h) simultanamente do lobus lateralis dexlev. processus candatus o processus papillaris do lobus crudatus. isto é, coictor do primeiro território e tronco formado por um contingente de cada região mencionada - 1 rez;

i) simultaneamente do lobus lateralis dexter. do lobus mediulis dexter e mocessus papilaris do lobus caudatus ou. melhor precisando, tronco composto por contribuiçăo do primeiro e do último território, mais eferente isolado do segunrlo - 1 vez.

O ducto resultante da junçāo do ramus luteralis lobi dextri e rumus processi cauduti, evidenciem-se todos eles livres de tributários ou nāo, é identificado, dentre as 47 peças $(94,0 \% \pm 3,4$ - Figs. 1, 2, 3, 4) componentes do grupo inicialmente referido, a constituir, 12 vezes $(24,0 \% \pm 6,0-$ Fig. 4), o próprio ramus principalis dexter. Nas restantes 35 preparaçōes $(70,0 \% \doteq 6,5$ - Figs. 1, 2, 3) o aludido ducto liga-se a outros coletores, sob diferentes combinaç๊es, a saber:

a) ao romus medialis lobi dextri, 22 veze's (Figs. 1, 2); a nova via bilifera assim formada recebe. $\mathrm{cm} 8$ das disseccôes (Figs. 1, 2), o ductus cysticus, nāo sem que antes, em 2 dos casos, se the tenha juntado tronco composto por três contribuições do lobus medirlis dexter (Fig. 1) e tronco oriundo da fusāo de três eferentes, vale dizer. dois deste lobo e um do lobus laterulis dexter;

b) ao coletor nascido da convergência do ramus medialis lobi dextri c ramus proressi papillaris, 6 vezes;

c) ao ramus processi papillaris, 5 vezes;

d) ao troneo integrarlo pelo ramus processi pripilloris a ramus lobi quadrati, 1 vez;

c) ao diectus rysticus, $1 \mathrm{vez}$.

Destes 35 figados, destacamos 24 - Figs. $1,2,3,41$, nos quais o coletor bilifero, constituído da mancira descrita, representa 0 próprio rumus principalis dexter.

Nos outros 3 casos $(6,0 \% \pm 3,4)$, que completam o total de 50 preparações, o ramus laterulis lobi dextri associa-se: 2 ve. zes $(4,0 \% \pm 2.8)$, a tronco resultante da fusāo do ramus processi caudati e ramus processi mpillaris; 1 vez $(2,0 \% \pm 2,0)$, a tronco para o qual convergem o ramus processi caudati, o rumus processi pupillaris c o ramus medialis lobi dextri. O ramus lateralis lobi dextri conserva sua independência no tocante à região drenada, 2 vezes, embora em 1 delas reccba o ductus cysticus; 1 vez, a ele chega contribuição procedente do processus caudatus do lobus caudutus. Nas mesmas 3 dissecçōes, o ramus mocessi cuudati, 2 vezes apresenta-se livre de afluentes; $1 \mathrm{vez}$ alia-se a tronco de dois coletores oriundos do processus papillaris do lobus caudatus e do lobus lateralis dexter. Livre ou não de tributários. o ramus urocessi cuuduti, compoe, 3 vezes, nova via bilifera com o ramus processi papillaris. Esta, vai reunir-se ao ramus lateralis lobi dextri 2 vezes, nāo sem que antes, em 1 dos casos, acolha contribuição do lobus mediulis dexter e 1 vez, associa-se ao ramus medialis lobi dextri.

2. Ramus medialis lobi dextri - O ramus medialis lobi dextri, que se incorpora 12 vezes $(24,0 \% \pm 6,0)$ ao sistema do ramus principalis sinister, surge, em 1 
FrRNANDFs FIIIO, A. - Contribuicano ao estudo das vias blliferas intra e extra-hepaticas

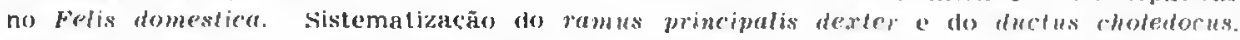

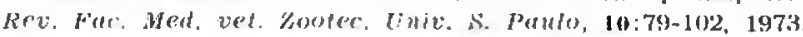

preparaçāo $12,0 \% \pm 2,0 \ldots$ Fig. 4), como raiz do duclus choledocus, após receber o ductus cystious; com mais elevada freqüencia, isto é, 37 vezes $(74,0 \%$ - 6.2 - Figs. 1, 2, 3), assinalamo-In, todavia, sob diferentes combinaçōes, a intograr o sistema do romus mincipulis dexter. Analisando este último grupo, descobrimo-lo, efetivamente. 16 vezes $(32.4 \% \pm 6,6-$ Fig. 1), a drenar somente o lobus mediulis dexter, embora dando também escoamento, em 3 peças, ao ductus cystirus. Nas mais $21(42.0 \% \pm 7.0)$, o rumus mediclis lobi dextri acolhe eferontes de outros territórios glandulares:

a) apenas do lobus quadrutus, 19 vezes, ou mais exatamente, um coletor - 14 vezes; dois ductos - 5 vezes; de realçar-se que, em 4 das peças, o tributário representa tronco de dois componentes;

b) exclusivamente do lobus lateralis dexter. um ducto - $1 \mathrm{vez}$

c) simultanoamente do lobres lateralis dexter, processus candatus do lobus caudatus e do lobus quadratus, isto é, coletor do último dos territórios mencionados e tronco formado pela fusão do dois eferentes do scgundo deles, mais um do primeiro - 1 vez.

Nestas 37 dissecções $(74,0 \%$-4: 6,2 Figs. 1, 2, 3), em que o ramus medialis lobi. dextri drena para o sistema do rumus principulis dexter, vêmo-lo aliado, sob diversos arranjos, às seguintes vias biliferas:

a) a tronco composto pelo rumus luteralis lobi dextri e rumus processi couduti -- 22 vezes - Figs. 1, 2); o novo contingente assim resultante, identificado livre de tributálios oriundos de outras regiỏes, 20 vezes - Fig. 2), embora acolha em 7 casns - Figs. 1, 2) 0 ductus cysticus recebe, antes deste, 1 vez - Fig. 1), contribuiçāo para a qual confluem três eferentes do lobus medialis dexter \&, 1 vez. afluente formado por dois coletores vindos do cilado lobo a mais um do lobre lateralis dexter:

b) ao remus processt pupillaris -.. 6 vozes - Fig. 3); no contingente assin constituido, lança-se. 1 vez. o ductus cysticus $c, 1$ vez, tronco para o qual concorrem contribuiçōes vindas do lobus culudratus, ou seja, uma do processus cuetdatus c outra do processus prpillaris;

c) a contingente composto, em seqüôncia, pela juncão do ramus lateralis lobi dex. tri, ramus processi cauduti e rumus processi papillaris - 3 vezes; o ramus medialis lobi dextri, esclareça-se, antes de participar da descrita associação, acolhe o ductus cysticus;

d) a tronco para o qual convergem por ordem, o rumus processi coudati, o romus processi pupillaris e o ramus lateralis lobi dextri - 2 vezes; o novo coletor formacio deste modo recebe. 1 vez, o ductus cysticus;

c) a contingente integrado, sucessivamento, pelo ramus processi cauduti, ramus lateralis lobi dextri e romus processi jupillaris - 2 vezes; o tronco assim surgido dá escoamento, $1 \mathrm{vez}$, ao ductus rystichs;

f) a troneo nuscido da confluência do ritmus processi cuududi o rumus processi prpillaris - 1 ve\%;

g) a contingente para onde drenam, pos ordem, agrupados dois a dois, o ramus laterulis lobi dextri o rumus processi caudati, de un lado, o ramus processi pupillaris e ramus lobi aluadrati, de outro -1 vez.

Fixaminadas as combinaçōes do ramus medirlis lobi dextri, no grupo das 37 pecas ora descritas, descobrimos que a via bilifera resultante das diversas associaçōes de coletores, configura, 25 vezes $150.0 \% \pm$ 7,1 - Figs. 1, 2), o próprio rumus princiwhis dexter; cabe ressaltar que, en 9 dis- 


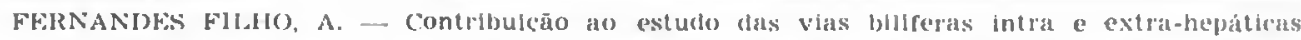

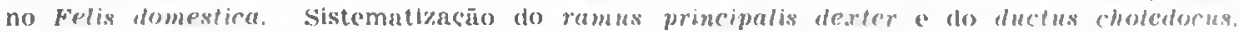

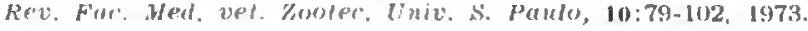

preparaçåo $(2,0 \% \pm 2.0$ - Fig. 4). como rai\% do ductus choledocus, após receber o ductus cystirus; com mais elovada froqüência, isto $\dot{e}, 37$ vezes $(74.0 \%-6.2$ - Figs. 1, 2, 3), assinalamo-Io, todavia, sob diferentes combinaçōes, a integrar o sistoma do ramus principulis dexter. Analisando este último grupo, descobrimo-lo, efetivamente, 16 vezes $(32,4 \%+6,6$ Fig. 1), a drenar somente o lobus medirlis dexter, embora dando também escoamento, em 3 peças, ao ductus cyslicus. Nas mais $21(42.0 \% \pm 7,0)$, o rumus medialis lobi dextri acolhe eferentes de outros territórios glandulares:

a) aprnas do lobius fucudrutus, 19 vezes, ou mais exatamente, um coletor - 14 vezes; dois ductos - 5 vezes; de realçar-se que, em 4 das peças, o tributário representa tronco de dois componentes;

b) exclusivamente do lobus lateralis dexter, um ducto -1 vesz;

c) simultaneamente do lobus luteralis dexter, processus cuudutus do lobus caudatus e do lobus quadratus, isto é, colotor do último dos territórios mencionados o tronco formado pela fusāo de dois eferentes do segundo deles, mais um do primeiro - 1 vez.

Nestas 37 dissecçōes $(74,0 \%$ 七 6,2 Figs. 1, 2, 3), em que o ramus mediulis lobi dextri drona para o sistema do remus wincipulis dexter, vêmo-lo aliarto, sob diversos arranjos, às seguintes vias biliferas:

a) a tronco composto pelo rumus lateralis lobi dextri e ramus processi cunduti - 22 veres - Figs. 1, 2); o novo conlingente assim resultante, identificado livre de tributários oriundos de outras regiōes, 20 vezes - Fig. 2), embora acolha em 7 casos - Figs. 1, 2) 0 ductus cysticus recebe, antes deste, 1 vez - Fig. 1), contribuiçāo j)ara a qual confluem três eferontes do lobus medialis dexter e, I vez, afluonte formado por dois coletores vindos do citado lobo mais um do lobus laterulis dexter:

b) ao ramus processi pupilluris - 6 veros ..- Fig. 3); no contingente assim constituido, lança-se, 1 vez, o ductus cysti(us e, 1 vez, tronco para o qual concorrem contribuiçōes vindas do lobus coudatus, ou seja, uma do processus candatus e outra do processus papillaris;

c) a contingente composto, em seqüencia, pela junçāo do rmmus lateralis lobi dextri, rumus processi cundati e rumus processi jotpillaris - 3 vezes; o rumus medinlis lobi dextri, esclareça-se, antes de participar da descrita associacão, acolhe o ductus rystirus;

d) a tronco para o qual convergem por ordem, o rumus processi candati, o ramus processi papilluris e o ramus lateralis lobi dextri - 2 vezes; o novo coletor formado deste modo recebe, 1 vez, o ductus cysticus;

(a) a contingente integrado, sucessivamente, pelo rumus processi culuati, rumus lateralis lobi dextri e ramus processi papillaris - 2 vezes; o tronco assim surgido dá escoamento, 1 vez, ao duefus rysticus:

f) a tronco nascido da confluência clo rumus processi comduti e rumus processi papillaris $-1 \mathrm{vez}$ :

g) a contingente para onde drenam, yor ordem, agrupados dois a dois, o remus lateralis lobi dextri e remuses processi cundati, de un lado, o ramus procossi unpllaris e rumus lobi yuadrati, de outro - 1 vez.

Examinadas as combinaçōes do remmus medialis lobi dextri, no grupo das $37 \mathrm{pe}$ cas ora descritas, descobrimos que a via bilifera resultante das diversas associaçōes de coletores, configura, 25 vezes $(50.0 \% \pm$ 7,1 - Figs. 1, 2), o próprio rumus principulis dexter; cabe ressaltar que, em 9 dis- 
FERNANDES FILIIO, A. - Contribuicão an estudo das vias biliferas intua e extra-hepaticas no Felis domestira. Sistematizacão do ramus principesis dexter e do ductus rholedorus. Ret. Har. Hed. ret. Zooter. Vinir. S. Paulo, 10:79-102, 1973.

seçōes - Figs. 1, 2). o ducfus (ysticus aparece como uma das raizes deste ramo.

3. Rumus proressi paprillaris, que integra o sistema clo ramus principulis sinister. rm 28 preparaçōes $(56,0 \%$ - 7,0- Figs 1. 2), apresenta-se conjugado ao ducelus hejuficus, 2 vezes (4.0\% + 2.8 ) e participa, nos mais 20 ligados $(40,0 \%$ $\pm 6.9-$ Fig. 3), do sistema do romus principulis dexter. Dentre as peças deste úllimo grupo, 16 vezes $132.0 \%$ 6,6), o rumus processi pupillaris escoa unicamente, o respectivo território glandular, ou seja, o processus propillaris do lobus reudulus. Nas restantes 4 disseceōes (8.0\% + 3.8), ( ramus processi papillaris recebe eforentes:

a) somente do processus caudatus do lobus caudatus, 3 vezes, isto $\dot{e}$, um contingente - 2 vezes o dois coletores 1 sez;

b) apenas do lobus quarlratus, uma contribuição 1 vez.

Veste último conjunto de 20 disseccôes. livere de afluentes vindos de outras regiōes glandulares ou encontrado a recebe-los, o rumus processi pupilluris reune-se às scguintes vias biliferas:

a) ao rumus medulis fobi dextri, 6 vezes -. Fig. 3); o coletor assim constituido, livre de tributários -- 5 vezes - Fig. $3)$, acolhe, em 1 peca, o ductus cysticus e. $\mathrm{cm} 1$ caso, contribuiçãn integrada por dois componentes oriundos do processus candatus do lobus caukatus e do processus pupillaris do lobus caudatus;

b) ao tronco onde se lancam o remus littermis lobi dextri o o ramus jrocessi caudati. 5 vezes;

c) ao contingente para o qual convergen o remps lateralis lobi dextri. o ramus processi cuuduli o o ramus medialis lobi dextri, 5 vezes; esclareca-se que. (m) 2 preparações no colotor então formado, desenboca o duclus rysticus; d) ro rumus processi canduti. 3 vezes; a contribuição assim resultante, acolhe, 1 vez eferente do lobus medialis dexter;

e) alo romus lobi quadrati. $1 \mathrm{vez}$

No conjunto das 20 peças ora descritas.o ramus processi papillaris, depois de associar-se às assinaladas vias, configura. 5 vozes $(10,0 \%$ 上 4,2$)$, coletor representativo do próprio romus principulis dexter; cumpre ressaltar-se que, 2 vezes, o ductus rysfiries configura uma das raizes reste ramo.

1. Ramus lobi cuadrati - O ramus lobi yuadrati, incorporado, $1 \mathrm{vez}(2,0 \%$ 卓 2,0), ao sistema do rumms principulis dexter e livre de tributários, une-se ao rumus proccssi pulllaris para formar contingente que se associa ao tronco constituido pelo rumus lateralis lobi dextri c ramus processi caudati; o novo coletor assim nascido mostra como afluente o ramus mediulis lobi dextri, a jusante do qual se converte no próprio romus principalis dextcr.

5. Ducfus cysticus - O ductus cysticus que, como se afirmou, integra. 16 veres $132,0 \%$ 上 6,6 - Fig. 3), o sistema do romus principulis sinister, drena com mair freqüência, is 10 c. 18 vezes $(36,0 \%$ \pm 6.8 Figs. 1. 2), para o sistema ro ramus mincipulis dexter. Também se antecipou que os 16 figados restantes. 11 vezes $(22,0 \%$ +t 5,8$)$ mostram-no a lançar-se no ductus heproticus; 3 vezss $(6,0 \%+3,4)$, a surgir, juntamente com os referidos ramos, como uma das três raizes do ductus choledocus; 1 vez $(2,0 \%$ + 2,0 Fig. 4), informe-se, cabe ao remus medialis lobi dextri, no qual so abre o ductus cysticus, esta condição. que é partilhada com o ramus principulis dexter e ramus principulis sinister e $1 \mathrm{Vez}(2,0 \%+2.0)$, elucide-st3. conpete an ramus lobi quadruti, ao qual chega o ductus cysticus, juntar-se ao ductus heputicus. 
FERNANDES FILIO, A. - Contribuicão ao estudo das vlas billeras intra e extra-hepalicas no Felis domestica. Sistematizacão do remus principalis dexter edo ducfus choledocus. Rev. Har. Meat. vet. Zouter". Ciniv. \$s. Paulo, 10:, , 1973.

Quando vinculado ao sistema do romus principulis dexter, o ductus cysticus sempre aparece livre de tributários, tendo sido descoberto a desembocar nas seguintes vias biliferas:

a) no tronco integrado pelo rumus lateralis lobr dextri, ramus processi cauduti e ramus medialis lobi dextri, 7 vezes Figs. 1, 2);

b) no ramus medialis lobi dextri, 4 vezes; o contingente entāo resultante acolhe, 3 vezes, por tronco, o ramiss laterulis lobi dextri, o rumus processi culduti e o ramus processi pupilluris e, 1 vez do mesmo modo, o rumus laterulis lobi dextri e o ramus processi caudati;

c) no coletor resultante da fusão do rumus lateralis lobi dextri, ramus processi caudati, ramus medialis lobi dextri e ramus processi papillaris, 4 vezes;

d) no tronco oriundo da união do ramus lateralis lobi dextri e ramus processi caudati. 1 vez;

c) no coletor para o qual concorrem o ramus medialis lobi dextri e rumus processi papillaris 1 vez; o novo contingente, assim formado, recebe contribuição identificada a drenar o ramus laterulis lobi dextri e o rumus processi candati;

f) no rumus lateralis lobi dextri, 1 vez; o tronco composto desta mancira recebo outro cle que participam o ramus processi cauduli. o rumus processi papillaris e o ramus medialis lobi dextri.

Elucidamos que. $\mathrm{cm}$ todas as peças enquadradas nas modalidades agora descritas. isto é, 18 vezes $(36.0 \% \pm 6.8$ - Figs. 1.2) os contingentes derivados das associações, tal como as discriminamos, constituem-se no próprio ramus principulis dexter.

6. Coletores inominados - As contribuicöes procedentes do lobus lateralis dexter, exclusive o ramus lateralis lobi dextri e respectivas raizes. contam-se, como afirmamos, ' $m$ número de uma -25 vezes $(50,0 \% \pm 7.1-$ Fig. 2) e de duas -5 veres $(10,0 \%+4,2)$; informamos que $\mathrm{m} 3$ das pecas constantes do penúltimo grupo, dila contribuicão ajuarece como tronco originado da convergência de dois componentes. Nas mais 20 disseccooes $(40,0 \% \pm 6.9)$ os coletores procedentes do lobus laterulis dexter, cingom-se, exclusivamente, an rumus luteralis tobi dextri e respectivas raizes.

Os ductos procedentes do lobus luterelis dexter antes de se lançarem $\mathrm{cm}$ colctores conspicuos unem-se, 10 vezes $(20,0 \% \pm 5.6)$ a contingentes:

a) do processus pupillaris do lobus caudatus, ou scja, a um - 4 vezes - Fig. 2); o coletor cntảo resultante endereça-se ao romus processi candafi - 3 vezes Fig. 2), ao tronco do rumus lateralis lobi dextri e ramus processi cundati $1 \mathrm{ver}$;

b) do processus candatus do lobus candelus, vale dizer, a um - 3 vezes, cum. prindo destacar que, em 1 das preparacōes, este contingente representa tronco de dois componontes; os coletores nascirios de lais associaçōes vão abrir-se - 2 vezes, na via bilifera para onde drenam o rumus lateralis lobi dextr e o rumus processi cunduli e, 1 vez, no ramus mediulis lobi dextri:

c) do lobus medistis dexter, melhor pre cisando, a um - 2 vezes sendo que em 1 peça, o eintingente constitui tronco de dois componentes; os coletores resultantes destinam-se a ducto formado pe. lo rumus laterulis lobi dextri a rumus mocessi candati - 1 ver e a outro composto polo ramms lateralis lobi dextri. ramus processi caudati c rumus medialis lobi dextri- $1 \mathrm{vaz}$;

d) simultaneamente, do processus caudatus e do processus pupilaris do lobus condalus, isto es dois coletores, por 
FERNANDES FILIUO, A. - Contribulçäo ao estudo das vias blliferas intra extra-hepaticas no Felis donfesticu. Sistematizacão do rainus prineipulis deater e do ductus choledocus.

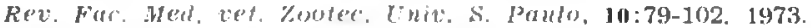

tronen 1 vez; a via assim integrada converge para contribuiçāo na qual se lançam o remus lateralis lobi dextri o o remus processi cuudati.

Nos restantes casos os eferentes do lobus lateralis acxire tom por destino direto

a) o romus processi randoti, 9 vezes, identificado a acolhor um colotor - $7 \mathrm{ro}$ zes a dois contingentes - 2 vezes;

b) o tronco para o qual concorrem o jamus laterulis lobi dextri e o rumus processi canduli, 12 vezes; com efrito, vimo-lo a receber um ducto - 11 vezos e dois colctores - $1 \mathrm{vez}$.

Quanto às contribuicões provenirntes do processus candatus do lobus candatus, afora o ramus processi cuuduli, surpreendemo-las, como já se descreveu, $\mathrm{cm}$ número de uma - 21 vozes $(42.0 \%+7,0)$ e de duas - $\ldots 5$ vezes $(10.0 \%+4,2)$; ressaltamos que em 2 pecas o colotor representa tronco de dois componentes. Nos restantes 24 figados $(48,0 \% \pm 7,1)$, as contribuicões escoadoras do processus cendufus do lobus cutudatus sāo representadas pelo próprio ramus processi comduti o respectivas raizes. Os eforontes inominados oriundos do processus caudatus do lobus comdatus, antes de se associarem a outras vias biliferas unom-se, 7 vezes $(14,0 \% \div 4,9)$ a contingentes vindos:

a) co lobus laternlis dexter. isto é, a un - 3 vezes; o nowo colctor dirige-se a tronco composto polo ramus lateralis lobi dextri e rumus processi caudati 2 vezes e an rumus medialis lobi dextri $-1 \mathrm{vez}$

b) do processus unpillaris do lobus caudatus, vale dizer, a um- 2 vezes; o ducto assim originado desemboca no troneo para o qual drenam o romus lateralis lobi dextri " o romus proressi caudali - 1 voz e no formado pelo remus me dialis lobi dextri o ramus processi pamillures - 1 ver; c) simultancamente, do lobus lateralis dexter e processus papillaris do lobus caudulus, ou seja, a tronco constituido por um eferente de cada território ․ 1 vez; 0 coletor rosultante vai ter ao ronco onde ste reunem o romus luteralis lobi dextri e o rumus processi cardati:

(1) Io lobis medialis dexter, melhor "sporrificando, a um - I vez; o ducto assinı surgido lanca-se no tronco composto polo romus lateralis lobi dextri e rumus proncessi cuuduli.

Nas outras preparaçoes os eferentes que escoam o processus cundutus do lobus caudatus atingem, dirrtamonte, as seguintes vias biliferas:

a) o Ironco comum ao ramus lateralis lobi dextri e rumus processi caudati, 15 vezes; efetivamente, descobrimo-lo a recrber um contingente - 13 vezes o duas contribuiçōes - 2 vezes;

b) o rumus processi pupilluris, 3 vezes: realmenie, encontramo-lo a acolher um coletor -2 vezes $e$ dois ductos -1 vez;

c) o ramies lateralis lobi dextri, a que choga um eferente - 3 vezes.

No tocante às contribuiçōes procedentes do lobus medialis dexter. excluido o rumus medialis lobi dextri o respectivas raizes, vimo-las, como foi assinalado, em número de uma $-(20,0 \% \pm 5,6-$ Fig. 1); esclarecrmos que, em 5 dos casos, tal via compreconde tronco de dois e dr três eromponentes. Nas restantes 40 pecas $(80,0 \% \pm 5,6)$, que totalizam o material estudado, os contingentes de drenagem do lobus mediulis dexter são representados pelo romus medicelis lobi dextri e respectivas raizes.

Os eferentes que derivam do lobus medialis dexter, antes de so lançarom a coletores mais calibrosos, associam-se 3 vezes 
FFRNANDES FILAO, A. - Contribuicăo ao estudo das vias biliferas jntra e extra-hepatloas

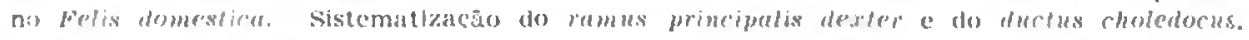

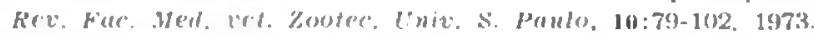

$(6,0 \% \pm 3.4)$ a outras contribuiçons originadas:

a) do lolus luterul's dexter. isto है. a uma - 2 veres; o ducto assim constituido enderecea-se a tronco formado pulá confluência do rimus laterelis lobi dextri e rumus processi cuuduli - 1 vez e a contingente nascido da fusão destes dois ramos mais o ramus mediulis lobi dextri - 1 vez:

b) do mocessus cruedatus do lobus caudetuss, vale dizer, a uma - $1 \mathrm{vez}$; o ducto resultante vai ter a tronco para o qual convergem o rumus laterulis lobi dertri co ramus processi cauduti.

Cos mais casos. os oferentes do lobus medirlis dexter desembracam diretamente:

a) no tronco integrado pelo romas laterulis lobi dextri o rumus processi cauduti. identificado a recolher um contingente - 4 rezes;

b) no rumus lateralis lobi dextri, visto a rcceber uma contribuiçã.o - 1 vez;

c) no coletor conum ao remus processi couduti o rumus processi pupilluris. (videnciado a acolher um ducto -1 ve\%;

(1) no contingente composto pelo remus luterulis lobi dextri, ramus processi coudenti a momus modialis lobi dextri. an qual $\mathrm{vem}$ tor um eforente - $1 \mathrm{vez}-$ Fig. 11.

Do tocante às contribuições oriundas de processus papillaris do lobus cuudutus, sem contar o ramus processi pupillaris a respectivas raizes, descobrimo-las, como se relatou, em número de uma - 10 veres $(20,0 \%$ $=5.6$ - Fig. 2); acrescentamos que. 2 vezes. o eforente representa tronco de dois componentes. Nias outras 40 disseçörs $180.0 \%+5.6)$ as vias de drenagem do processus pepilluris do lobus coudutus sāo representadas pelo rumus processi impillaris e respectivas raizes.
Os ductos nascidos no lobo en apreço, antes de atingirem contingentes conspicuos, reunem-se a coletores de outros territórios, a saber:

a) do lobus lateralis dexter, ou soja, a um - 4 vezes - Fig. 2); o ducto então originado dirigr-se ao rumus processi cuuduti - 3 veres … Fig. 2) e a tronco para o qual confluem o romus lateralis lobi dextri a o rumus processi caulati-- 1 ves;

b) do processus comdulus do lobus candatus. melhor dizendo. a um -2 vezes; o contingente resultante desemboca no troneo que drena o ramus lateralis lobi dextri e romus processi cunduti -1 vez e no formado polo ramus medialis lobi dextri e rumus processi papillaris - 1 vez:

c) simultanoamente. do lolus lateralis dexter c processus coudutus do lobus cundutus. vale dizer. com a via bilifera surgida da reuniāo de dois contingentes - 1 vez; a contribuicão assim integrada abre-se no troneo composto pelo rumus loferalis loli dextri e romus processi rumblati.

Nas pecas restantes os coletores vindos do processus pupilluris do lobus cendutus afluem diretamente para o remmes processi cunduti - 2 veres e no tronos formado pelo rumus laterulis lobi dextri a romus processi combli - 1 vere.

No que diz respleito a coferentes do lobus ynudratus, exclusive o ramus lobi quedruti e respectivas raizes. contam-se, como referimos. un - 14 vezes $(28,0 \%+6.3) e$ dois - 6 vezes $(12.0 \%$-4,6); aduzimos que tais eferentes, em 4 casos, representam tronco de dois componentes. As focadas vias de drenagem não foram. aliás. vistas a estabelecer associaçöes com as de outros terrilórios. isto o. lançam -se diretamente nos seguintes ductos de maior calibre:

a) no ramus medialis lobi dextri. 20 vezes; de fato, descobrimo-lo a receber um co- 
FERNANDES FILHO. A. - Contribuicāo ao estudo das vias biliferas intra e extra-hepáticas no Fulis domestica. Sistematização do ramus principalis dexter e do ductus choledocus. Rev. Fac. Hed. vet. Zooter, Univ. S. Paulo, 10:79-102. 1973.

letor - 15 vezes e dois contingentes 5 vezes;

b) no ramus processi papillaris, idontificado a acolher uma contribuição - 1 vez.

As 30 preparaçōes $(60.0 \%$ I 6.0), que completam o total de 50 , exibem os eferentes do lobus quadratus a drenar para o romus lobi quadrati, na condição de raizes.

7. Constituiçāo do romus principalis dexter - Como verificamos, o sistema do ramus mincipulis dexter surge, no estudado conjunto de 50 pecas, compreendendo a associação dos seguintes ductos conspicuos: romus lateralis lobi dextri, ramus processi cundati, ramus medialis lobi dextri, ramus processi prupillaris, ramus lobi quadruti e ductus cysticus o sistema do ramus principalis derter drona. ainda, contingentes inominados. oriundos do lobus lateralis dexter. do processus caudatus do lobus caudatus. do lobus medinlis dexter, do processus papillaris do lobus caudatus e do lobus quadratus.

Assim, especificando, nos 50 fígados, 0 ramus mincipalis dexter apresenta a seguinte composição:

a) ramus latcralis lobi dertri e ramus processi candati - 12 vezes - Fig. 4);

b) ramus lateralis lobi dextri e ramus processi couduti, integrando tronco para o qual converge o ramus medialis lobi dextri - 9 vezes;

c) ramus laterntis lobi dextri e ramus processi cauduti, formando tronco a que se aliam, primeiro. o ramus medialis lobi dextri e, depois. o ductus cysticus - 7 vezes - Figs. 1. 2);

d) ramus lateralis lobi dextri e ramus processi comdati, conjugados em tronco para o qual conflue outro, integrado pelo ramus medialis lobi dextri c ramus processi papillaris - 5 vezes - Fig. 3); e) rumus laterulis lobi dertri e ramus processi cuudati. fundidos em tronco que recebe. sucessivamente, o ramus processi papillaris e o ramus medialis lobi dertri, onde se abre o ductus cysticus - 3 vezes;

f) remus lateralis lobi dextri o ramus processi caudali reunidos em tronco, ao qual se juntam. primeiro, o romus medialis lobi dextri e a seguir o ramus processi pupilluris - 3 vezes;

g) agrupamento identien at anterior, com a participação final do ductus cysticus - 2 vezes:

h) ramus Intcralis lobi dextri e ramus processi cauduti convergentes para tronco a qur se associa o ductus cysticus - 1 vez;

i) ramus lateralis lobi dextri c rumus processi caudati, constituindo fronco no qual desembocam, primeiro, via bilifera resultante da confluência do mamus lobi yuudruti e rumus processi papillaris e. por fim. isolado, o rumus medialis lobi dertri - 1 vez:

j) rumus luterulis lobi dextri e ramus processi cauduti, formando tronco a que se aliam. inicialmente. também associados, o rumus medialis lobi dextri e o ramus processi papilluris e. a jusante deles, o ductus cysticus - 1 vez;

1) ramus luterulis lobi dextri c rumus processi caudali, conjugados em tronco visto a acolher, um apos outro, o ramus processi pupillaris e o rumus medialis lobi dextri - 1 vez;

m) agrupamento identico ao anterior, com a contribuiçāo final do ductus cysticus - 1 vez;

n) ramus processi caudati c ramus processi mepillaris, fundidos em tronco para o qual afluem, independente. o ramus medialis lobi dextri e, por via bilifera comum. o ramus lateralis lobi dextri e o ductus cysticus - 1 vez; 


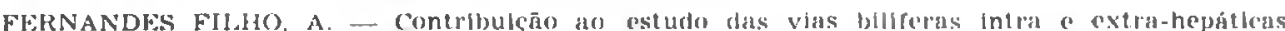

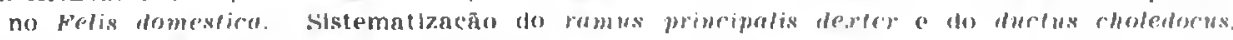

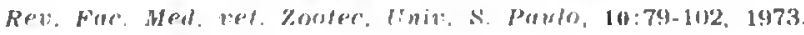

(3) ramus latermlis lobi dextri e romus processi couduti, reunidos om tronco. onde se lança o romus mediulis lobi dextri de que e tributário o ductus rysticus - 1 ver:

p) rumus processi comdati e ramus processi papilluris, associados $\mathrm{em}$ tronco para qual concorrem, sucessivamente, o ramus laterulis lobi dextri e o ramms medialis lobi dextri - 1 vez:

q) agrupamento idêntico ao antruior, com a integraçāo final do ductus cysticus .1 ver.

Nascido das combinaçoes ora descritas, o rumus princimalis dexter, livre, 45 veres $190,0 \%$ 七 4.2 - Figs. 1. 2. 3. 4) recerbe nos mais 5 figados $(10,0 \%+4.2)$, contribuiçoes procedontes:

a) lobus laterulis derter, isto c. uma 2 vezes; esclareca-se que om 1 das pe. cas, o coletor representa tronco de dois componentes;

b) simultaneamente, do lobus laterulis der. ler processus condutus do bolus cunditus, 2 vozes, melhor procisando, um contingente de cada território - 1 ver um do primeiro e dois do segundo ... I vez;

c) do propessus candatus do lobus comdalus. vale dizer, uma, constituida por três eferontes - 1 vez.

Na composição do ramus principalis dexterr. em machos e fêmeas, a intervençăo do ramus mediatis lobi dextri e ramus proressi pupillares fa\%-se segundo poreentagens, cuja diferenca não é estatisticamonte significante. para $\alpha=5 \%$.

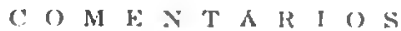

Parecenos conveniente iniciar a discussão dos nossos resultados, confrontando-os com os de JABLAN-PIANTTIC $\overline{0}$ (1963) os de NETTFLBLAD * (1954). (mmbora apenas o primeiro dos $\Lambda A$. se tenha valido dos métodos de investigação que adotamos, ou seja, os de dissecçào "radiológico; a rigor, a visada comparação o bastante favorecida pelo fato de havermos empregado a mesma nomenclatura de que ele se serviu, cm oposiçāo à utilizada por NFTTFLBLAD (1954), cujos dados, além do mais, se funclamentam no exame de pecas preparadas por corrosain. De outra parte, as observacōes colhidas nos trabalhos de OTTAVIANI" (1933) \& BRESSOU \& VLADUTIU: (1944), estabelecidas, por aquele, somente $\mathrm{cm}$ bases radiológicas e, pelos dois últimos, com auxilio de disseccōes, corrosōes a radiografias, afiguram-sc-nos bastante genéricas para permitirem comparaça mais proveitosa. pois. nenhum deles objetiva a sistrmatização das vias biliferas intra e extra-hejáticas nos moldes a que nos propusemos; inclusive, nem se valeram da mesma terminologia anatomica. Quanto aos tratadistas, as poucas e resumidas informacôe. encontradas, bem podem ser inseridas no corpo dos comontários que faromos a rospeito das contribuiçōes dos AA. precedente mente citades.

Com relação aos ductos hepatocisticos. cumpre logo esclarecer, apesar de assinalacks por LESBRE' li (1922). MARTIN (1923). FILINIBERGER \& BAUM + (1932) ( BOURDEILLF: \& BRFSSOU ' 1953), nåo os descobrimos, tal como sucedeu a JABLAN-PANTIC :5 (1963), NETTELBLAL ) (1954), OTTAVIANI" (1933), BRFSSOU \& VLADUTIU: (1944), REIGHARI) \& JFNNINGS 11) (1935) \& DAVISON (1947). () deslize prende-se, de modo provável, à orienlação seguida pela maioria dos AA. de anatomias vetrrinárias, que, muitas vezes, inarlvertidamente, estendèm às várias espécjes domésticas conhecimentos correspondentes a um animal padrūo; quem sabe, o tratamento conjunto aplicado, a canideos e felideos teria concorrido, também, para a falha. pois, a existencia de ductos hepatoristicos em cãrs, í conhereida. 
FERNANDES FIJIIO, A. - Contribulẹa au estude das vias bilferas intra c extrn-hepáticas no Felis domesticu. Sistematizacāo do ramus principalis dexter e do ducfus choledrocus.

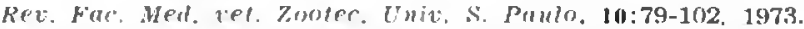

Outrossim, merecé atençāo, de imediato, o exame do durtus cysturus. que identificamos, na majoria das veros $(36,0 \%)$, a lancar-se no sistema do romus principulis dexler, fato igualmente referido por NETTELBLAD * (1954). Contraditam-no JABLANPANTIC: (1963) \& BRESSOU \& VLADUTIU = (1944). para os quais o citado ducto, rospectivamonte, abre-se com maior freqüncia no duclus heputicus e representa uma das três raizes do duclus choledorus. comportamintos por nós verificados, respeifarla a sequiência, cm $22.0 \%$ e $6.0 \%$ das pe(as. Alude-se, aincla, a menor freqüência com que o ructus cysticus desemboca no durtus hepatious a no rumus pricipulis siwister ou no ramus principalis dexter, ramus principulis sinister o romus medialis lobi dextrit. As preparaçoes que fizemos, entrotanto, mostram o ductus rysticus. se conccituado à mancira leste A., afluindo: 16 vezes $(32,0 \%)$, para o sistema do ramus principalis sinister; 13 vezes, para o rumus principulis dexter; 5 vezes, para o ramus mediulis lobi dextri; 1 vez, para o rumus lateralis lobi dextri e $1 \mathrm{vez}$ para o romus lobi culudrati. A propósito, resta-nos acusar a ausência, em nosso materkal, do arranjo notado na única radiografia exibida por OTTAVIANI:" (1933), qual seja, o da união do ductus cysticus ao conduto do lobo direito, nascido, a adotar-se a nomenclatura que perfilhamos, da confluência do rumus luteralis lobi dextri e do ramus mediulis lobi dextri.

No tocante ao durfus choledorus, assin consicierado. por MARTI.N i (1923) ELLENBERGER \& BAUM : (1932), a jusante da chegada do último tributário, respretivamente, ao prolongamento do ducto principal e ao ductus cysticus, JABLANPANTIC 5 (1963), REIGHARD \& JEN.NINGS 10 (1935) \& DAVISON \& (1947) dizem-no constituido pelo ducto hepático principal (ductus heputicus) to ductus cysticus, disposiçāo que, comprovada por nós diversas vezes $(22,0 \%)$. s'gundo se adiantou, é definicla pelo primeiro doles como a mais co- mum. Menos precisamente, BOURDELLF \& BRFSSOU' (1953) descrevem o ductus choledocus a surgir da uniāo do canal cislico (ductus cysticus) a coletores hepáticos. Aliás. BRESSOU \& VLADUTIU: (1944). expressandr-se com certa liberalidade, assinalam o ducto colédoco a resultar da fusāo dos canais hepáticos esquerdo e direito (ramus principulis sinister a romus principulis dexter, da nomenclatura que seguimos). $\mathrm{cm}$ rujo ângulo de convergência chega o ducto cistico; de fato, no esquema da distribuição das vias biliferas, por eles apresentado, nāo se evidencia a insinuada triplice convergència, pois, esse ducto é visto a lancar-so no canal hepático esquerdo. Como se antecipou, a triplice confluência do romus principutis dexter, romus principulis sinister e durtus cysticus, também documentada em desenho por JABIAAN-PANTIC" (1963), ocorre em $6,0 \%$ dos nossos casos. O arranjo mais frequentemente observado nas preparações que fizemos, entretanto. corresponde à origem do ductus choledocus por duas raizes, vale dizer, o ramus princiunlis dexter e o romus principalis sinister $(68.0 \%)$; duas modalidades são ainda identificadas, raramente, ou seja, a da sua constituição por triplice convergência, envolvendo, as ora citadas vias e o rumus mediulis lobi dextri $(2,0 \%)$ e a da sua formacão à custa de duas raízes, o duclus hepaticus e o rumus lobi cuudruti (2.0\%). Alguns esquemas destinados, por JABLAN-PANTIC 5 (1963), a registrar as variaçōes dos ductos biliferos, no gato. parecem deixar dúvidas quanto à afirmativa genérica do A., quando escreve que. nos carnivoros, o ductus choledocus nasce. mais comumente. da junçāo do durtus hepaticus an ductus rystirus.

s: ainda JABLAN-PANTIC (1963) quem afirma tor encontrado, por vezes, ramos secundários do processus pupillaris a atingir diretamente. o ductus choledocus, disposi(ầo por nós nảo assinalada; ao contrário, em nosso material rogistra-se, apenas 1 vez. contribuicaào procedente do lobus lateralis simister al alcançar o focado ducto. 
FERNANDES FItIO. A. - contribuicano ao estudo das vias buferas intra e extrathepations

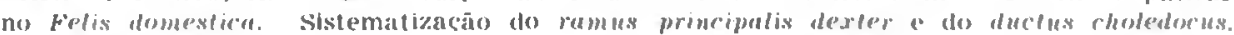

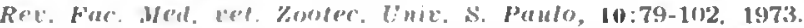

Entrando agora a aupreciar o problema da sistemalização do rumus mincipulis dexrter, destacamos, de inicio, os achados coincidentes de JABLAN-I'ANTIC: (1963) e Nl:TTELBLAD * (1954) (a nomenclalura adotada por este A. vai entre parêntesis) quando afirman que os coletores oriundos do lobus lutemlis dexter lobus dorsocuadulis dexter), do mocessus cuudatus do lobus culudatus (jurers dorsulis dexter do lobus medius dorsalis) e do lobus mediulis dexter (lobus medius ventrulis dexter) drenam para o rumus prinripulis dexter. por intermédio, obedecida a ordem de enumeração. do ramus lateralis lobi dextri (mmus dorsocandulis dexter), do ramus processi cunduti (ramus dorsalis dexter) a in ramus medialis lobi dextri cramus ventrorraniulis dex(er), comportamento por nós verificado somente em $18.0 \%$ das disseccōes. OTTAVIAVI" (1933) aponta o conduto bilifero direito (rumus principulis dexter) a derivar da reuniāo de dois afluentes vindos dos lobos direito (conduto do lobo direito) $\mathrm{c}$ caudato (conduto do lobo caudato); estas vias. a saber, o tronco formarlo, cono se nota na única radiografia documental, prolo ramus laterulis lobi dextri a ramus medialis lobi dextri e maio o rumus processi reudati, isolado, assin nāo qualificados no texto pelo A., nela aparecem como tais; ressalte-se" que esta disposiçăo nāo foi encontrada em nossas peças. Aliás. sógundo BRESSOU \& VLADUTIU = (1944), o canal hepático dirojto (rumus princibulis dexter) resulta da convergência dos troncos superior e inferior. responsáveis polo escoamento, correspondentemento, do lobo caudato (na realidade proressus ruudutus do lobus ruudutus) e lobo direito (lobus laterulis derter), de um lado e clo lobo internediário direito (lobus medialis dexter), de outro. Desta forma, deduzimos, o tronco superior apresenta duas raizes, ou seja. o romus processi coudati e o rumus lateralis lobi dextri o o inferior. apenas uma, o próprio rumus medialis lobi dextri. Enfim, consoante RFIGHARD \& JFNNINGS 10 (1935), os dois ou mais co- letores endereçados ac) ducto cistico, independentes ou nāo, nasoom, um, supomos, o ramus principulis dexter, da junção d"s pesquenos cofrentes do lobo ristico robus medialis dexterb. do ambas as divisōes do lobo lateral direito clobus luterulis dexter) e do lobo caudato eprocessuss cendatus do lobus crudalus). Insistimos no fato de que a conposicão global do rumus principulis dexter, regisitada por BHFSSOU \& VLADUTIU:- (1944) e REIGHARD \& JENNINGS 11 (1935). foi demonstrado em $18.0 \%$ dos nossos casos; quase outros tantos (16,0\%), exibem a integrar o sistema do romus principulis dexter, além dos já moncionados ramos, o ductus rystirus. Fxaminando os exquemas de JABLAN-PANTIC 5 (1963), verifica-so que, na maioria das vezes, o ramus latemulis lobi dextri e o rumus processi culedeti concorren, exclusivamente ou não, para a formaçāo do ramus prinripulis dexter: aliás, o primeiro dos arranjos, assinalamo-lo f'm $24.0 \%$ das disseceôes. Dele pouco difere a disposição caracterizada pola presenca de tronco resultante da fusáo dos citados ramos, visto a receber o ductus rysticus, como descobrimos, raramente $12.0 \%)$. Bem mais comum é encontrar-se o romus principulis dexter dando escoamonto ao rumus laterulis lobi dextri, ramus processi coudali, ramus mediulis lobi dextm o rumus precessi papillaris. ou identificar-se cslos ramos associados ao ductus cystucus, comportamentos notados, respectivamente, em $20,0 \%$ ( $18,0 \%$ das peças que mrepramos, Enfim, nosso material exibe, excepecionalmente, as enumeradas vias to remus lobi quadrati a drenarrm para o rumus principulis dexter $(2,0 \%)$

Torna-se oportuno, agora, analisar os várins tipos de associaçāo dos coletores, destacando, de inicio, que o romus laterulis lobi dextri e o ramus proxessi randali compōem. em 94.0\% dos figados, a raiz mais regularmente constituida do romus principalis de $x$ ter: os integrantes desta raiz, podem drenar. unicamente, os respectivos territórios glandulares $(62,0 \%)$ ou, antes de se unirem, acolher. sob diferentes combinaçöes, efe. 
FERNANDES FIIIIO, A. - Contribuicăo ao estudo das vias biliferas intua e extra-hepaticas

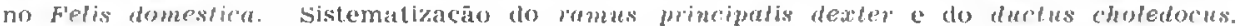
Rev. Fac. Wed. vet. Foolec. Lniv. S. Paulo, 11:79-102. 1973.

rentes de outros lobos $(32,0 \%)$, consoznte discriminamos, minuciosamento, ato fornecer os resultados. Nos restantes $6.0 \%$, o romus lateralis lobi dextri. livres ou não de 1ributários, junta-se ao tronco formado polo mums processi cauduli a ramus processi pupillaris $(4,0 \%)$ o, ar mascido da confluêneia clos dois precedentes, mais o rumus medialis lobi dextri $(2,0 \%)$; nas $m-s m a s$ disseccoues. o romus processi cenduti, isolado ou nāo. funde-se com o ramus processi papilleris $(6,0 \%)$ indo a nova via bilifera reunir-se ao remus laterulis bobi dextri $(1.0 \%)$ a a ramus medialis lobi dextri $(2,0 \%)$. Na radiogralia do trabalho de OT"IAVIANI" (1933), notamos o concluto do lobo caudato trumus frocessi rendali) a lanģar-se no conduto do lobo direito (romus lesteralis bo bi dextri e rambs medialis lobi dextri, conjugados), mas o A. Lambém o descreve a dosombocar no ducto hrpático, arranjos não verificados em nosso matrial. Para BRFsSOU \& VIAADUTIU 2 (1944), o tronco superior (uma das raizes do canal hepatico direito - romus mincipulis dexter), recothe bile dos lobos direito e caudato, comportamento também observadido por NET'TFL$\mathrm{BLAD}^{2}$ (19504) e JABLAN-PLANTIC 5 (1963).

Quanto ao romms medialis lobi dextri. por nós assinalaclo a participar do sistrma do romus principulis sinister $(24,0 \%)$ a a figurar como uma clas raizes do ducfus choledocus (2,0\%), surge, (om $74,0 \%$ rlas disseccörs, entre as vias biliferas constituintes do rumus primoipelis dexter, quer descoborto a drenar somento o lobus medialis deater $(32.0 \%)$, quer combinato a coletores oriundos de outras regiōes glandulares $(12.0 \%)$, consoante especificados no itrm 2 do capitulo Resultados. As associaçoes do remers medialis lob: dextri, que registramos, ditem resprito, por ordem de froqüencia: ao (ronere composto pelo remus lateralis lobi deatri a moms processi enudeli (22 vezes); an romus processi pupillaris (6 vezos); ao contingente integraclo pelos tres coletores ora reseridos (3 veress); a tronco para o qual convergem o remess processi canduti, o rumus processi pupillaris o o ramus lateralis lobi dextri (4 veres) o a contingentes resultantes da confluência do romus proressi conduti e romus procossi mpilloris (1 vez) ou da reuniano do romus lalerwlis lobi dextri. rumus processi rendati, ramus proressi pupillaris e ramus lobi quadruti 11 ver). A junçāo do ramus mediatis lobi dextri romus lateralis lobi dextri, denons1 rada por (OT"TAVIANI! (1933) (n) radiografia. nän ocorre nas nossas peças; a possibilidade de uniāo do troneo do remus let teralis boli dextri a romms processi conduli itroneo superior) ao ramus modialis lob: fextri (tronco inferior), acusada por BRESSOU \& VIADUTIU: (1944), também a verificamos (22 vezos), como já a haviam documentado NFT'THLBLAD's (1954) (sob diferente sinonimia) (JABLAN-PLANTIC" (1963).

Com rolacão an remus processi popilluris. por nós identificado, mais comumente, a drenar para o sistema do rumus principalis sinister $(56.0 \%)$ e. em carátor exceucional. a lancar-se no ductus heputicus $(4,0 \%)$, encontramo-lo, com elevada frcqüencia, isto é, em 40,0\% dos casos a representar uma das vias biliferas incluidas no sistema do rumus zrincipalis dexter, seja a escoar apemas o provessins pruilleris do lobus cuudafus $(32.0 \%)$. seja aliado a eferentes originados em outros territörios glandulares $(8,0 \%)$, como elucidamos no item 3 do capitulo Resultados. As associagōos do ramses froresisi jupillaris envolvem. por ordem de freqüência: o romus madialis fobi dextri (6) vezes); tronco constituido pelo ramms loferelis lobi dextri e rumus processi cunthet (5 varos); contingente integrado pelos romus laterulis lobi dextri. romus processi

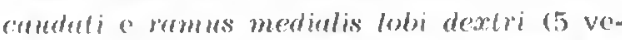
zes); o romus processi fanduti (3 veros) a

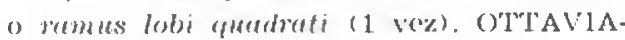
NI" (1933), BRLSSOU \& VIAADUTIU * (1944) \& NH'ITHIBLAD s (1954) não aluacm a coletor que, procortente do processus pupillaris do lobus culudulus, venha a integrar o sistema do ramus mincipolis dexter; 
FERNANDES FILHO. A. - Contribuicão ao estudo das vias blliferis intra extra-hepaticus $n$, $F$ its domestica. Sistematiacão do ramus principalis dexter edo ductus choledocus Rev. Fac, Med, vet. Zorter. Univ. S. Paulo, 10:79-102, 1973.

() contrário sucorde a .JABLA.N-PLANTIC: (1963), pois, dentro os esquemas por cle exibidos, um doles revela-nos tal particifaçāo.

No atinente an rumus lobi cuadrati, incorporado ao sistema do rumus principulis dexter em $2,0 \%$ das dissecçōes que realizamos, livre de tributários, associa-se an wmus processi papilluris formando tronco; a este une-se outro, constituido pelo ramus laterulis lobi dextri e ramus processi cauduti. Segundo OTTAVIANI:" (1933), afluente primário do lobo quadrado corre, excepcionalmente para a direita, disposição a que JABLAN-PLANTIC is (1963) alude, afirmando: no gato, de modo especifico, chegam. às vezes. ao rumus principalis dexter. o ramus lobi quadrati a o rumus medials lobi sinistri; para BRESSOU \& VLADUTIU: (1944) e NETTEI.BLAD * (1954), todavia, o principal colctor desse lobo smpre faz parte do sistema do rumus princiunlis sinister. Dentre as vias biliferas componentes do sistema do romus principulis dextor. nāo nos coube doparar com o rumus medict. lis lobi sinistri, fato notado por JABLANPLANT.C: (1963)

A respeito do ductus rysticuss, assinalado cm nosso material. como se afirmou, a integrar o sistema do remes principulis sirister $(32,0 \%), e$, o do romus principulis dexter $(36,0 \%)$, também o descrevemos a lancar-se no ductus licyaticus $(22,0 \%)$ e a configurar uma das raizes do ductus choledorus $(6,0 \%)$. Indicanos, igualmente, os (asos em que o ductiss rysticus pode associar-so ao romus modialis lobi dextri $(2,0 \%)$ (" romus lobi quadmali $(2,0 \%)$, quando $\mathrm{cs}-$ tes sāo vistos a represontir, lespectivantente, raiz do ductus choledorus e tributário do ductus heprlicus. Quando vinculados ao sistema do romus principulis dexter. sonum? livere de afluentes, o dectus cysticus abro-se, consoante ajuramos, no tronco composio polo ramus lateralis lobi dextri, ramus processi cruadati a ramus madialis lobi dextri $(7$ vozes); no rumus medialis lobi dextri (4 vezrs); no contingente resultante da fusão do rumus laterulis lobi dextri, romus processi candati c ramus processi mulla- ris (4 vezes); no tronco oriundo da união) do romms laterulis lobi dextri e romus processi reludeti (1 ver); no contingente para o qual concorrom o remus mediulis lobi dextri e ramus processi papillaris (1 vry) c no ramus lateralis lobi dextri (1 vez). Na documentação ajresentada por OTTAVIANIs (1933), o ductus cysticus desemboca. aparentemente, no conduto do lobo direito (tronco formado polo ramus leterelis lobi dextri a ramus medialis lobi dextri); no entender de BRESSOU \& VLADUTIU * (1944), entretanto, esso duclo vai ter ao ângulo definiclo pelos canais hepáticos direito (romus prineipolis derter) e esquerdo (rimus principalis sinister). Torna-se, assim, impossivel comentar tais achados, pois, os do primeiro A. não encontram corresponclência $\mathrm{em}$ nossas disseccooes e os do segundo, pecam pela imprecisão clas informaçōes fornocidas. Para NETTELBI,AD * (1954), o duclus cysticus lança-se no rumus ventrocruniatis dexter (ramus medialis lobi dextri), no rumus principulis dexter, no ductus hepaticus ou no remus mincipulis sinister, arranjos que coincidem com os irientificados por JABLAN-PANTIC (1963); aliás, a terceiua destas modalidades. igualmento reforida por RFIGHARD \& JFNNINGS 10 (1935) C DAVISON * (1947), descobrimo-la em 22,0\% das disseccôes: Quanto aos outros compertamentos, fritas as devidas ressalvas, tamberm as confirmamos no material de que dispusemos; assim. no focante à primeira delas (5) veges), somento podemos dizer flos casos relativos à participaçāo do remus medial is lobi dextri no sistema lo ramus primcipulis aver (4) vezes) ou da sua apresentaçäo como raiz do duretus choledorms (1 vez); a segunda (13 vezes), so adotado o criterio de JABL.AN-PANTIC: (19)63), ocorteu do mesmo modo, cm várias das nossas peças; a propósito da quarta $(32,0 \%)$, recordamos - caráter genérico da informação por nós prestada, pois, limitamo-nos a csturlar a constituiçäo do ductus choledorus e lo reftmus princiunlis dexter. 


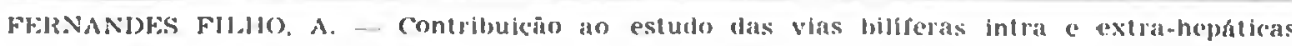

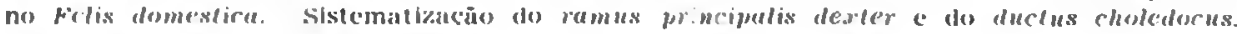

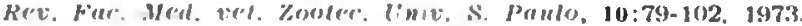

Consideranclo-se, por finı, a oriontação que nos traçamos, qual soja. il de sistomatizar os coletores inominades do bolus latemolis dexter. do proressus roudutus do lobus comedolus. do lobus mediulis dexter. do proressus pupbllaris do lobmes renulatus os do lobus youdrulus, am termos do associação, rxcluidas as vias do drenagem para o sisloma do ramus principulis sinister, a a de especoificar a composicano do remmus principulis dexter, reportamo-nos apenas as informaçōes contidas nos jtens 6 a 7 do (atpifulo Rosultados, poiss, nènhum comentário recolhemos, a resproito, na literatura compulsada. Con base nesses dacios, dostaque-se que as associaçoes de colctores inominados, procodentes de lerritórios distintos, fa\%m-so sempre dirotam nte să incomuns; já as e'slabclecidas, querr dircta. quer indiretamente. ontre tais coiletores o as vias biliferas principais, surgen com clevada freqüencia, graças sobretudo as relacours que envolvem contingentes: do fobus luterulis dexter a lobus condulus cprocessus cundulus), do lobus modialis dexter a lobus rundralus, do lobus candentus (processus comblus) e lobus medialis dexter, do lobus laterulis dexter e lobus malialis dexter. ainda, do lobus luterulis derter a lobus cundulus (processus pupilluris).

Cumpre, também, ressallar a presença constanto do rumus lulerulis lobi dextri o romus processi rauluti, na composiçäo do rumus prinripulis dexter, de que participam. ainda, o romus modialis lobi destri, e.m grando numero de observaçōes; o remus proressi pupilloris, am pouco monos da motade delas o o romuss lobi yuendruti. rarissimamente. Einfin, o duetus cystiress, confirmamos, drena para os sistemas do romus primeipalis dexter a momes primeipulis sinislep, segundo porecentigens quase rquivitlentess.

\section{O N C I, L S S s}

Fstudando as vias biliferas intra " extrahepáticas que dronam para o ramus principulis dexter, b(rm como a particiunçăo dos- te ramo o cle scus tributários na constituição do ductus choledoress, com base na dissceção de 50 figados de gatos domésticos. sem raça definida, 29 machos (23 adultos ( 6 jovens) e 21 fémeas $(17$ adultas e 4 jov('ns), julgamos válidas, a partir dos resultados colhicios, as conclusōes adiante disriminarlas.

1 - O) ductus choledorus que, em 34 perças $(68.0 \%)$. resulta da fusảo do romms principulis dexter o ramus principulis sinister. o visto lambén, o por ordom de freqüência, a originar-se da reuniào do ductus hepulirus ao duclus cystirus, 11 vezes (22,0\%); da triplice coníluéncia do remus mincipulis dexter, romus principulis sinister a remus medialis lobi dextri, 1 vere $(2,0 \%)$ e da junçäo do duatus heputirus o ramus lobi anudrati. 1 vee $12.0 \%)$.

2 - Isirre k0 lributários, em 49) casos $198.0 \%)$. somente 1 ve\% $(2.0 \%) 0$ ductus cholvelorus acolhe coletor procedente do lobus lateralis sinister.

3 - () ductus cysticus integra-se, em 18 disserçoes $(36,0 \%)$, ao sistema do ramus mincipulis dexter. tenclo sido idontificado a lançar-se. 7 vezes $(14.0 \%)$, no troneo formado polo ramus lateralis lobi dextri. remus mocessi conduti a ramus mediulis lobi dextri: a vezes $(8,0 \%)$, no rumus mediulis lobi dextri: 4 vezes $(8.0 \%)$. no coletor resultanto da fusāo do rumus laterulis lobi dextri, rumus processi coludati, ramus medialis lobi alextri o ramus processi pupilloris; $110 \%(2,0 \%)$, no contingonte oriun(lo) dì uniāa do rumus lateralis lobi dertri no ramus proressi cumdati: 1 ve\% $(2,0 \%)$, no troneo para o qual eoneorrem o romus medialis lobli arolvi o rumus processi puwilluris: 1 leog $(2,0 \%)$, no romus laterelis lebi dextri.

4 - O ductus cystirus. quer dreno para o sistena do rumus principulis dexter, fato verificalo 18 vezes $(36,0 \%)$, quer represento", juntamento com o ramus principulis dexter a romus primeipulis sinister, uma das 


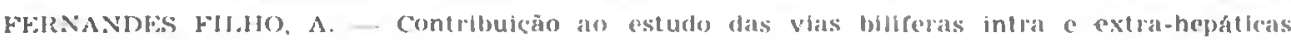

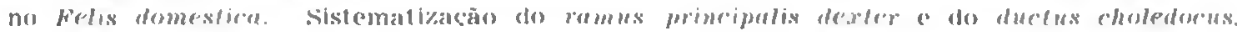

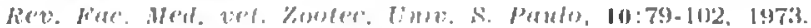

três razes do durtus choledorus, disposi(अäo encontrada 3 vores $16.0 \%)$. mostlit-so livre de tributários.

5 - O rumus principulis dexter lem por raizes, 12 vozes (24.0\%), o romus luterolis lobi dextri o o romus processi comadati: 10 vezes $120.0 \%$ ). estres dois contingentes, mais o ramns mediulis lobi doxtri e o romus processi pupillaris: 9 vezes (18.0\%), o rumus luteralis lobi dextri, o rumus mocessi cunduri o o ramus mediulis lobi dextri: 9 veres $(18.0 \%)$, estes três colclores. mais o rmmus mocessi pupilluris e o durtus cysticus; 8 vezss $(16.0 \%)$, o romus luteralis loli dextri, o rumus processi coundui, o romus medialis lobi dertri e o ductus cysticus; 1 ve\% (2.0\%), o rumus lateralis lobi dextri, o rumms processi cunduti o o ducfus cysticus: $1 \mathrm{ve}$ (2.0\%), o romus laterutis lobi dextri. n rumus processi cuuduti. o rumus mediulis lobi dextri. o remus promessi pupillaris a o ramus lobi quendreati.

6 - Una ve\% individualizarlo, o romus primeipulis dexter exibe-se live de tributários 45 vezes $(90.0 \%) \quad c$, recobn coletores vindos do lobus luteralis dexter, 2 vezes $(1,0 \%)$; simultaneamente, do lobms foteralis dexper e do mocesises comdentus do lobus creudalus, 2 voress $(4,0 \%)$; clo moressus raudelus do lobus coudulus, 1 ver $(2,0 \%)$.

7 - Con exclusão do rumus luterulis lobi dextri. do romus prosessi canduti, do rammes mediutis lobi dextri, do remus procossi pmpillaris, do ramms lobi amodrali do duelus cystir:us, sompre ünicos, surpreendomos variável numoro de afluentes ino. minarlos, que se integlim ale sistema (lo) remus primeipulis dexter, ou seja. do lobes lateralis dexter, um, 25 veres $(50,0 \%)$ e dois,

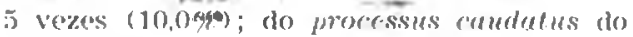
lobus comblus, um, 21 vezes (12.0\%) ( dois. 5 vezes $(10.0 \%)$; do lobus medintis dexter, um, 10 vozes $(20,0 \%)$; do processus mupilleris do tobus cuudutus, um. 10 vezes $(20,0 \%)$; do lobus chudrutus, un, 14 vezes $(28,0 \%)$ ( dois, 6 vezes $(12.0 \%)$.
8 - Na constituicano do romus mincipalis dexter, não foram registratas diferençs estatisticanento significantes. quando confrontados os sexos.

RF MV-A 11

Frasiddes Fin, A. - Contribution to the stady of the extrahelutio and intrahemefie bile alerts in rats. Sistematization of the ramus principalis dexter and the ductus cholodocus. Rav. Fre. Menl. vit. Zooter. 【niv. S. Paulo, 10: 79-102, 1973.

SLIMMARY - It wus studied, ing dissecution, the systemalization of the ramus principalis dexter and the ductus choledocus in 5) cots.

The system of the ramus principalis dexter is composed, mainly, by the ramus latoralis lobi dextri, ramus processi caudati. ramus medialis lobi dextri, ramus processi papillaris, ramus lobi quadrati and ductus cysticus.

The ductus choledocus is integrated by the ramus principalis dexter omd ramus principalis sinistor $(68.0 \%)$, by the ductus hepaticus and ductus cysticus (22.0\%), by the ramus principalis dextor, ramus principalis sinistor and ductus cysticus $(6.0 \%)$, by the ramus principalis dexter, ramus principalis sinister and ramus medialis lobi dextri (2.0\%), by the ductus hepaticus and ramus lobi quadrati $(2,0 \%)$.

UNITERMS - Bile durels*; Crels*

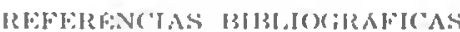

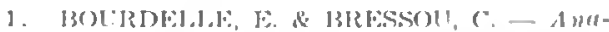

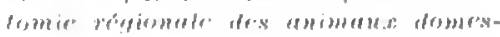

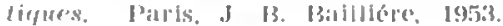
p. $3(1) 2-9) 3$.

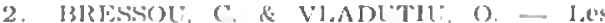
allores, les velnes ol les fanaux blliares intla-hopatlques she\% le chat. Rec. Merl. let., 1:0:16i1-67, 1911.

3. 1)AVISON, ᄉ. Mammalian anntomy. 7." ed. Philadelphia. The blakiston ('o. 19.17. p. 165 . 


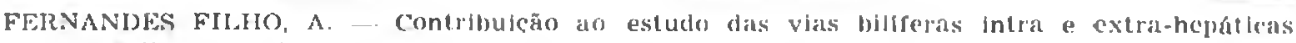
no felis domestica. Sistemalloacāo do rames principatis derter e do ductus choledorus.

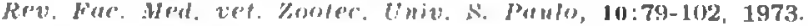

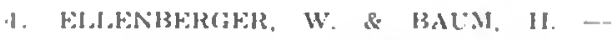

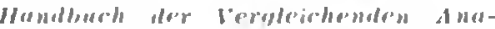
tomie der Hanstiere. 17 Aur. Borlin, Julius springer, 19:32. p. 170.

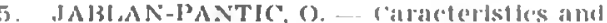
compatative gatios of intrabopatic bile duct in comestic animils. Aclu ver. frelur. 13:3-13, 1063.

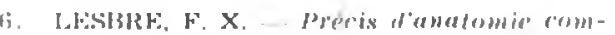

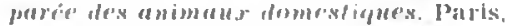
J. B. Biallliere, 1922, v. 1, D. 6ti5.

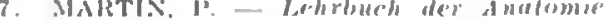
der Hunstieres. Stuttgart, Sohlekhardt \& Holser, 192:3. v. I. I. 217
\&. NFtTheibIAI), S. C. - Die I.obiorung und Innere "Iopographice dej Siaugerlobor. deta Anrt., (based), 21 isuspit. 20): 3-251, 1951 .

9. OTTAVIANI, (i, - lRicerche radiografiche, comparative sulle vie hilifere intraepallene. Alli lst. veneles sci. mal. " naf., 9:: : 106511:27, 1933.

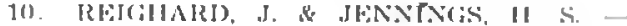
Anefomy of the cat. 3." ed. . New rork, llenry llodt and Co., 19:35, 1) 2.41.

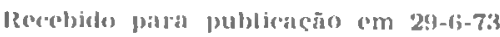
Aprovadu pala publicaç̄o om 6i-7-73

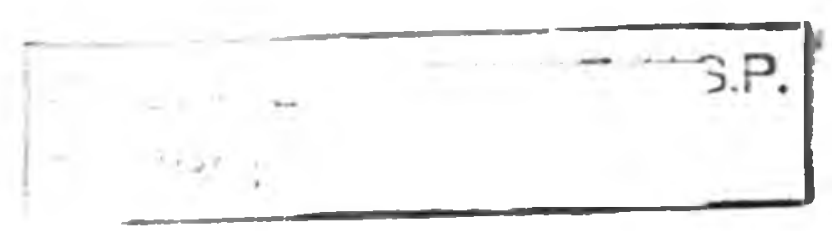

